Original Research Paper

\title{
A Comparison of Explicit Semi-Analytical Numerical Integration Methods for Solving Stiff ODE Systems
}

\author{
${ }^{1,2}$ E.R. El-Zahar, ${ }^{1,2}$ H.M. Habib, ${ }^{3}$ M.M. Rashidi and ${ }^{2}$ I.M. El-Desoky \\ ${ }^{1}$ Mathematics Department, Sciences and Humanities College, Salman bin Abdulaziz University, Alkharj, 11942, KSA \\ ${ }^{2}$ Basic Engineering Science Department, Engineering Faculty, Shebin El-Kom, Menofia University, Egypt \\ ${ }^{3}$ Shanghai Automotive Wind Tunnel Center, Tongji University, Jiading, Shanghai 201804, China
}

Article history

Received: $15-03-2015$

Revised: $27-05-2015$

Accepted: 20-06-2015

Corresponding Author:

E.R. El-Zahar

Department of Mathematics,

Sciences and Humanities

College, Salman Bin Abdulaziz

University, Alkharj, 11942,

KSA

Email: essam_zahar2006@yahoo.com

\begin{abstract}
In this study, a comparison among three semi-analytical numerical integration algorithms for solving stiff ODE systems is presented. The algorithms are based on Differential Transform Method (DTM) which are Multiple-Step DTM (MsDTM), Enhanced MsDTM (EMsDTM) and MsDTM with Padé approximants (MsDTM-P). These methods can be classified as explicit one step semi-analytical numerical integration methods. The error and stability analysis of each method is presented. New important relationships among the methods are introduced. To demonstrate our results, a comparison of the accuracy, stability and computational efficiency of the methods is presented through solving some linear and nonlinear problems arising in applied science and engineering.
\end{abstract}

Keywords: Multiple-Step Differential Transform Methods (MsDTM), Enhanced MsDTM, Padé Approximants, Stiff Problems, Accuracy, Stability

\section{Introduction}

Many mathematical problems arising from the real world cannot be solved completely by analytical means. One of the most important mathematical problems arising in applied science and engineering is stiff ODE systems. Stiff ODE systems, which have significantly different timescales of change, occur in many fields of engineering particularly in the studies of electrical circuits, chemical and biochemical reactions, optimal control, vibrations and fluid mechanics; see for example (Schiesser, 1993; El-Zahar, 2015). The problem of stiffness has been known for some time and has attracted the attention of many numerical analysts leading to surveys of methods for stiff problems. It is well known that explicit numerical methods are intrinsically faster than commonly used implicit methods while implicit methods are commonly used for solving stiff problems because of their stability (Lambert, 1991; Hairer and Wanner, 1996; Butcher, 2008). In fact, the explicit numerical methods which recently are presented in $(\mathrm{Wu}, 1998$; Wu and Xia, 2001; 2007; Novati, 2003; Ahmad et al., 2004; Ahmad and Yaacob, 2005; Ebady et al., 2012; Egbako and Adeboye, 2012; El-Zahar, 2013a; El-Zahar et al., 2014a) can also satisfy the stiff problems.

Recently, semi-analytical numerical methods such as Homotopy Perturbation Method (HPM), Variational
Iteration Method (VIM), Differential Transform Method (DTM) and Adomian Decomposition Method (ADM), are applied for finding approximate analytical solutions for a wide classes of nonlinear problems. Solving stiff problems is one of the most recent applications of these methods; see for example (Mahmood et al., 2005; Guzel and Bayram, 2005; Darvishi et al., 2007; Hassan, 2008; Zhao and Xiao, 2010; Aminikhah and Hemmatnezhad, 2011; Aminikhah, 2012; Zou et al., 2012; Atay and Kilic, 2013; Zhao et al., 2014; El-Zahar et al., 2014b). However, for some important classes of problems such as stiff ODE problems, singularly perturbed problems, chaotic and non-chaotic problems and nonlinear oscillators and for the sake of large convergence region, accuracy and efficiency, it is necessary to treat each of the above mentioned semi-analytical numerical methods as an algorithm in a sequence of time intervals. Therefore, different multiple-step HPM, DTM, ADM and VIM are presented for solving many important classes of problems; see for example (Adomian et al., 1988; Mahmood et al., 2005; Ghosh et al., 2007; Goh et al., 2008; Chowdhury et al., 2009; 2012; 2014; Gonzalez-Parra et al., 2009; Alomari et al., 2010; Wang et al., 2010; Odibat et al., 2010; Molli et al., 2013; Keimanesh et al., 2011; Gokdogan et al., 2012a; 2012b; Yildirim et al., 2012; Erturk et al., 2012; El-Zahar 2012a; 2012b; Do and 
Jang, 2012; Alquran and Al-Khaled, 2012; Lee et al., 2013; Patra and Ray, 2013; Smarda et al., 2013; Heydari et al., 2014; Momani et al., 2014; Olvera et al., 2014; El-Zahar, 2015).

Although the treatment of HPM, ADM and VIM as multiple-step methods speeds up the convergence rate and improves the accuracy, these methods have some limitations which hamper their applications.

An analytical integration is required for each step, each solution series term in HPM and ADM and each iteration in VIM and so some difficulties may arise as follows:

- The nonlinear and non-homogeneous parts of the ODE may be ill-conditioned such that the integration became very complicated

- By increasing the number of iterations or the number of solution series terms, the number of terms of approximate solution may increase, so rapidly that the integration becomes both complicated and time consuming

- "In VIM for some differential equations, we may have more than one Lagrange multiplier. Let $L_{1}$ and $L_{2}$ be different Lagrange multipliers for an ODE. We assume that $L_{1}$ is more accurate than $L_{2}$ and then by using $L_{1}$ the more fast the approximation to the exact solution can be obtained, but the computation will be complicated. The mentioned notation for $L_{2}$ is totally opposite" (Heydari et al., 2013; 2014)

- In ADM, for nonlinear problems, we need to evaluate the Adomian polynomials that mostly require tedious algebraic calculations

- In HPM, we should suitably choose an initial guess, or infinite iterations are required

Due to the above mentioned difficulties, the Multiple-step DTM (MsDTM), which does not require analytical integration, evaluation of the Lagrangian multiplier, or rather difficult computation for finding the Adomian polynomials, is one of the most effective multiple-step semi-analytical numerical methods.

EL-Zahar, (2015) presented an adaptive step size MsDTM for solving singularly perturbed ODE systems, also known as stiff ODE systems. The results showed that the proposed method is an accurate and efficient method compared to classical MsDTM and RK4 method. However, for large values of the admissible local error or when a fixed step size is used, the problem of stability arises. A studying of this obstacle and how to overcome it is introduced in this study through presenting a comparison of the stability and accuracy of three semianalytical numerical differential transform based methods. These methods are MsDTM, Enhanced MsDTM (E-MsDTM) and MsDTM with Padé approximants (MsDTM-P). These methods can be classified as explicit one step semi-analytical numerical methods. The stability and error analysis of each method is presented. New important relationships among these methods are introduced. To demonstrate our results, a comparison of the accuracy, stability and computational efficiency of the methods is presented through solving some linear and nonlinear problems of practical importance in applied science and engineering.

\section{Multiple-Step DTM}

The basic definition and the fundamental theorems of the MsDTM are given in (Odibat et al., 2010; Keimanesh et al., 2011; Gokdogan et al., 2012b; Yildirim et al., 2012; Erturk et al., 2012; El-Zahar, 2012b; Khader and Megahed, 2014). For convenience of the reader, we present a review of the method as follows.

Consider the following non-linear initial-value problem:

$\frac{d y}{d t}=f(t, y(t)), y\left(t_{0}\right)=C_{0}$

Let $\left[t_{0}, T\right]$ be the interval over which we want to find the solution of the initial value problem (1). In actual applications of the DTM, the Nth-order approximate solution of the initial value problem (1) can be expressed by the finite series (Zhou, 1986; Nik and Soleymani, 2013; Rashidi et al., 2013; 2014; Al-Amr, 2014; Benhammouda et al., 2014):

$$
y(t) \approx \sum_{k=0}^{N} Y(k)\left(t-t_{0}\right)^{k}=S(t), \quad t \in\left[t_{0}, T\right]
$$

where:

$$
Y(k)=\frac{1}{k !}\left[\frac{d^{k} y(t)}{d t^{k}}\right]_{t=t_{0}}
$$

Equation (2) and (3) imply that the concept of differential transformation is derived from the Taylor series expansion. The following theorems can be deduced from (2) and (3)

\section{Theorem 1}

$$
\text { If } y(t)=\beta(u(t) \pm v(t)) \text {, then } Y(k)=\beta U(k) \pm \beta V(k)
$$

Theorem 2

$$
\text { If } y(t)=u(t) v(t) \text {, then } Y(k)=\sum_{\ell=0}^{k} U(\ell) V(k-\ell) .
$$

Theorem 3

$$
\text { If } y(t)=\frac{d^{m} u(t)}{d t^{m}}, \text { then } Y(k)=\frac{(k+m) !}{k !} U(k+m) .
$$




\section{Theorem 4}

If $y(t)=(\beta+t)^{m}$, then $Y(k)=\frac{H_{m, k} m !}{k !|(m-k)| !}\left(\beta+t_{0}\right)^{m-k}$, where $H_{m, k}=\left\{\begin{array}{ll}1, & \text { if } \quad m-k \geq 0 \\ 0, & \text { if } \quad m-k<0\end{array}\right.$.

\section{Theorem 5}

$$
\text { If } y(t)=e^{\lambda t}, \text { then } Y(k)=\frac{\lambda^{k}}{k !} e^{\lambda t_{0}}
$$

\section{Theorem 6}

$$
\text { If } y(t)=\sin (\omega t+\beta) \text {, then } Y(k)=\frac{\omega^{k}}{k !} \sin \left(\omega t_{0}+\beta+\frac{k \pi}{2}\right) .
$$

\section{Theorem 7}

$$
\text { If } y(t)=\cos (\omega t+\beta) \text {, then } Y(k)=\frac{\omega^{k}}{k !} \cos \left(\omega t_{0}+\beta+\frac{k \pi}{2}\right) \text {. }
$$

Using some fundamental operations of DTM, we have the following recurrence relation:

$$
Y(k+1)=F(k, Y(k)), Y(0)=C_{0}
$$

where, $F(k, Y(k))$ is the differential transform of the function $f(t, y(t))$. The differential transform $Y(k)$ of the unknown function $y(k)$ can be obtained by solving the iterating algebraic system (4). In order to speed up the convergence rate and to improve the accuracy of resulting solutions, the entire domain $\left[t_{0}, T\right]$ is usually split into sub-intervals and the algorithm of MsDTM is applied as follows:

Assume that the interval $\left[t_{0}, T\right]$ is divided into $M$ subintervals $\left[t_{m}, t_{m+1}\right], m=0,1, \ldots \ldots . M-1$ of equal step size $h=\left(T-t_{0}\right) / M$. The main ideas of the MsDTM are as follows. First, we apply the DTM to Equation (1) over the interval $\left[t_{0}, t_{1}\right]$ to obtain the following approximate solution:

$y_{0}(t) \approx \sum_{k=0}^{N} Y_{0}(k)\left(t-t_{0}\right)^{k}=S_{0}(t), t \in\left[t_{0}, t_{1}\right]$

Using the initial conditions $y_{0}\left(t_{0}\right)=C_{0}$. For $m \geq 1$ and at each subinterval $\left[t_{m}, t_{m+1}\right]$ we will use the initial conditions $y_{m}\left(t_{m}\right)=S_{m-1}\left(t_{m}\right)$ and apply the DTM to Equation (1) over the interval $\left[t_{m}, t_{m+1}\right]$, where $t_{0}$ in Equation (5) is replaced by $t_{m}$. The process is repeated and generates a sequence of approximate solutions $y_{m}(t)$, $m=0,1, \ldots \ldots . M-1$, for the solution $y(t)$ :

$$
y_{m}(t) \approx \sum_{k=0}^{N} Y_{m}(k)\left(t-t_{m}\right)^{k}=S_{m}(t)
$$

Finally, the MsDTM assumes the following solution:

$$
y(t)=\left\{\begin{array}{lc}
y_{0}(t) \approx S_{0}(t), & t \in\left[t_{0}, t_{1}\right], \\
y_{1}(t) \approx S_{1}(t), & t \in\left[t_{1}, t_{2}\right], \\
\vdots & \\
y_{M-1}(t) \approx S_{M-1}(t), & t \in\left[t_{M-1}, t_{M}\right]
\end{array}\right.
$$

\section{Error Analysis of MsDTM}

The local truncation error of the approximate solution $S_{m}(t)$ is estimated by the inequality (Do and Jang, 2012; Bervillier, 2012; El-Zahar, 2013b):

$$
\begin{aligned}
& \left\|y_{m}(t)-S_{m}(t)\right\| \leq C \frac{\left(t-t_{m}\right)^{N+1}}{(N+1) !}, C=\max _{\xi \in\left[t_{m}, t_{m+1}\right]}\left|y_{m}^{(N+1)}(\xi)\right|, \\
& t_{m} \leq t \leq t_{m+1}, m=0,1, \ldots \ldots, M-1
\end{aligned}
$$

It is well known that stiff ODE systems are associated with the existence of eigenvalues with very large magnitudes which consequentially results in solutions usually having exponential behavior.

Therefore, if we let $C=K^{N+1}$, in Equation (8), where $K=\max _{\xi \in\left[t_{m}, t_{m+1}\right]}\left|y_{m}^{(1)}(\xi)\right|$, then Equation (8) results in:

$$
\begin{aligned}
& \left\|y_{m}(t)-S_{m}(t)\right\| \leq \frac{\left[K\left(t-t_{m}\right)\right]^{N+1}}{(N+1) !}, K=\max _{\{t, y\} \in R}|f(t, y)|, \\
& t_{m} \leq t \leq t_{m+1}, m=0,1, \ldots \ldots, M-1
\end{aligned}
$$

From the local truncation error (8), it is clear that, for a fixed order $N$, as the step size $h=\left(t-t_{m}\right)$ tends to zero the approximate solution $S_{m}(t)$ tends to the exact solution $y_{m}(t)$ and thus the method is consistent, while numerically, from (9), for a fixed step size, the convergence of the MsDTM only begins when $N$ is of order $O(K h)$.

\section{Stability Analysis of MsDTM}

In order to examine the MsDTM for the stability, let us consider the differential equation:

$y^{\prime}(t)=\lambda y(t)$

where, $\lambda$ is a complex constant and $\operatorname{Re}(\lambda)<0$. For this equation, the MsDTM solution, Equation (6), yields:

$S_{m}\left(t_{m+1}\right)=\sum_{k=0}^{N} Y_{m}(k) h^{k}$ where $h=t_{m+1}-t_{m}$

Form Equation (10) we have $k ! Y_{m}(k)=\lambda^{k} Y_{\mathrm{m}}(0)$ and $Y_{m}(0)=S_{m}\left(t_{m}\right)$, then we get:

$S_{m}\left(t_{m+1}\right)=\left[\sum_{k=0}^{N} \frac{(h \lambda)^{k}}{k !}\right] S_{m}\left(t_{m}\right)$ 
Setting $z=\lambda h$ in Equation (12) results in the amplification factor:

$$
R_{N}(z)=\sum_{k=0}^{N} \frac{z^{k}}{k !}
$$

Which is the $N^{\text {th }}$ order Taylor expansion of $\mathrm{e}^{\mathrm{z}}$ and thus the MsDTM is not A-stable method.

\section{Enhanced MsDTM}

Since the approximate solution obtained by MsDTM may yield an increasing error due to the nonlinearity (Do and Jang, 2012), a modification of MsDTM, which is called Enhanced MsDTM (Do and Jang, 2012) is presented as a multi-stage integration method as follows:

Suppose that we have solved the problem (1) up to a point $t_{m-1}, m=1,2, . ., M-1$ and have obtained a value $\mathrm{S}_{\mathrm{m}-1}\left(t_{m}\right)$ as an approximation of $y_{m-1}\left(t_{m}\right)$. Assuming the localization hypothesis (Lambert, 1991), $y_{m}\left(t_{m}\right)=\mathrm{S}_{\mathrm{m}}$ ${ }_{1}\left(t_{m}\right)$, we are interested in obtaining an approximate value for the true one $y_{m}\left(t_{m+1}\right)$. For that purpose, the following method is developed.

The solution $y_{m}(t)$ verifies the following IVP:

$$
\frac{d y_{m}}{d t}=f\left(t, y_{m}(t)\right), y_{m}\left(t_{m}\right)=S_{m-1}\left(t_{m}\right)
$$

Equation (14) can be written as:

$$
y_{m}(t)=S_{m-1}\left(t_{m}\right)+\int_{t_{m}}^{t} f\left(\tau, y_{m}(\tau)\right) d \tau
$$

We can replace $y_{m}(\tau)$ in Equation (15) by the approximate solution $S_{m}(\tau)$ from Equation (6) to obtain an improved approximation $S_{m}^{1}(t)$ to $y_{m}(t)$ :

$$
S_{m}^{1}(t)=S_{m-1}\left(t_{m}\right)+\int_{t_{m}}^{t} f\left(\tau, S_{m}^{0}(\tau)\right) d \tau
$$

where, $S_{m}^{0}(\tau)=S_{m}(\tau)$ and thus we can update the value $S_{m}\left(t_{m+1}\right)$ by:

$S_{m}^{1}\left(t_{m+1}\right)=S_{m-1}\left(t_{m}\right)+\int_{t_{m}}^{t_{m+1}} f\left(\tau, S_{m}^{0}(\tau)\right) d \tau$

Then, $Y_{m+1}(0)=S_{m}^{1}\left(t_{m+1}\right)$ from Equation (17) instead of $Y_{m+1}(0)=S_{m}\left(t_{m+1}\right)$ from Equation (6). For each subinterval, repeat this process, Equation (17), to update the initial condition of the followed subinterval, $Y_{m+l}(0)$ and consequently to obtain enhanced approximation to $y_{m+l}(t)$.

The above process, Equation (17), is called Enhanced MsDTM with one iteration ( $\left.\mathrm{E}_{1} \mathrm{MsDTM}\right)$. To apply Enhanced MsDTM with two iterations, we solve the integral equation, Equation (16) and replace $S_{m}^{0}(\tau)$ by
$S_{m}^{1}(\tau)$ to get $S_{m}^{2}(t)$. Repeating this successive approximations $I$-times by replacing $S_{m}^{I-1}(\tau)$ by $S_{m}^{I}(\tau), I=$ 1,2,3.. results in enhanced MsDTM with $I$ iteration ( $\left.\mathrm{E}_{\mathrm{I}} \mathrm{MsDTM}\right)$ as follows:

$$
\begin{aligned}
& S_{m}^{I}(t)=S_{m-1}\left(t_{m}\right)+\int_{t_{m}}^{t} f\left(\tau, S_{m}^{I-1}(\tau)\right) d \tau, \\
& I=1,2,3, \ldots \ldots, m=0,1, \ldots \ldots, M-1
\end{aligned}
$$

and hence we get an enhanced initial condition $Y_{m+1}(0)=S_{m}^{I}\left(t_{m+1}\right)$.

In fact the above process, Equation (18), is a multiple-step successive approximations method and it is equivalent to multiple-step Picard iteration method (Djang, 1948; Lal and Moffatt, 1982; Wazwaz, 2011) with DTM solution as the zeroth approximation of $y_{m}(t)$.

\section{Error Analysis of E-MsDTM}

It is well known that (Innocentini, 1999; Youssef and El-Arabawy, 2007) when the function $f(t, y(t))$ satisfies Lipschitz condition with respect to $y(t)$ in a rectangle

$R, R=\left\{\left|t-t_{m}\right| \leq a,\left|y(t)-S_{m-1}\left(t_{m}\right)\right| \leq b\right\}$, $|f(t, y(t))-f(t, \bar{y}(t))| \leq L|y(t)-\bar{y}(t)|, L=$ constant, then, irrespective of the choice of the initial approximation, the successive approximations $S_{m}^{I}(t)$ converge on the interval $\left[t_{m}, t_{m} \pm \min \left(a, \frac{b}{H}\right)\right], \quad H=\max _{\{t, y\} \in R}|f(t, y)|$ to the solution of the problem (1). Also, the error of the approximate solution $S_{m}^{I}(t)$ can estimated as follows:

$$
\begin{aligned}
& \left\|y_{m}(t)-S_{m}^{I}(t)\right\| \leq L \int_{t_{m}}^{t}\left\|y_{m}\left(\mu_{1}\right)-S_{m}^{I-1}\left(\mu_{1}\right)\right\| d \mu_{1} \\
& \leq L^{2} \int_{t_{m}}^{t} \int_{t_{m}}^{\mu_{1}}\left\|y_{m}\left(\mu_{2}\right)-S_{m}^{I-2}\left(\mu_{2}\right)\right\| d \mu_{2} d \mu_{1} \\
& \leq L^{I} \int_{t_{m}}^{t} \int_{t_{m}}^{\mu_{1}} \ldots \ldots \int_{t_{m}}^{\mu_{I-1}}\left\|y_{m}\left(\mu_{I}\right)-S_{m}^{0}\left(\mu_{I}\right)\right\| d \mu_{I} \ldots . . d \mu_{2} d \mu_{1} \\
& \leq C L^{I} \frac{\left(t-t_{m}\right)^{N+I+1}}{(N+I+1) !}, \leq C L^{I} \frac{\left(t-t_{m}\right)^{N+I+1}}{(N+I+1) !} \\
& \leq \frac{C}{L^{N+1}} \frac{\left[L\left(t-t_{m}\right)\right]^{N+I+1}}{(N+I+1) !}, C=\max _{\xi \in\left[t_{m}, t_{m+1}\right]}\left|y_{m}^{(N+1)}(\xi)\right|, \\
& t_{m} \leq t \leq t_{m+1}, m=0,1, \ldots \ldots ., M-1
\end{aligned}
$$

It is clear that the choice of the initial approximation, $S_{m}^{0}(t)=S_{m-1}\left(t_{m}\right)$, i.e., $N=0$, results in the following bounded error: 


$$
\begin{aligned}
\left\|y_{m}(t)-S_{m}^{I}(t)\right\| & \leq \frac{C}{L} \frac{\left[L\left(t-t_{m}\right)\right]^{I+1}}{(I+1) !}, C=\max _{\xi \in\left[t_{m}, t_{m+1}\right]}\left|y_{m}^{(1)}(\xi)\right|, \\
& \leq \frac{H}{L} \frac{\left[L\left(t-t_{m}\right)\right]^{I+1}}{(I+1) !},
\end{aligned}
$$

which is the classical Picard iteration method error (Youssef and El-Arabawy, 2007).

From the local truncation error (19), it is clear that, for a fixed number of iterations $I$, as the step size $h=(t-$ $\left.t_{m}\right)$ tends to zero the approximate solution $S_{m}^{I}(t) \rightarrow y_{m}(t)$ and thus the method is consistent, while numerically, for a fixed step size, the convergence of $\mathrm{E}_{I}$-MsDTM only begins when $I$ is of order $O(L h)$.

\section{Stability Analysis of E-MsDTM}

In order to examine the stability of E-MsDTM with $N^{\text {th }}$ order and $I$ iterations, we consider again Equation (10).

Using $\mathrm{E}_{1}-\mathrm{MsDTM}$, the approximate solution of Equation (10) is given by:

$$
S_{m}^{1}(t)=S_{m}^{0}\left(t_{m}\right)+\int_{t_{m}}^{t} \lambda S_{m}^{0}(\tau) d \tau
$$

where, $S_{m}^{0}(t)$ is the zeroth approximation of $y_{m}(t)$ and given by:

$$
S_{m}^{0}(t)=\sum_{k=0}^{N} Y_{m}(k)\left(t-t_{m}\right)^{k}
$$

From Equation (21) and (22) we have:

$$
S_{m}^{1}(t)=S_{m}^{0}\left(t_{m}\right)+\sum_{k=0}^{N} \lambda Y_{m}(k){\frac{\left(t-t_{m}\right)^{k+1}}{k+1}}^{k}
$$

Since $(1+k) Y_{m}(k+1)=\lambda Y_{m}(k)$ for Equation $(10)$, then Equation (23) becomes:

$$
S_{m}^{1}(t)=S_{m}^{0}\left(t_{m}\right)+\sum_{k=0}^{N} Y_{m}(k+1)\left(t-t_{m}\right)^{k+1}
$$

and can be written as:

$$
S_{m}^{1}(t)=\sum_{k=0}^{N+1} Y_{m}(k)\left(t-t_{m}\right)^{k}
$$

which is the $(N+1)^{\text {th }}$ order approximate solution of (10) obtained by DTM. Replacing the solution in Equation (22) by that obtained in Equation (25) and repeating the above algorithm $I$ times results in:

$$
S_{m}^{I}=\sum_{k=0}^{N+I} Y_{m}(k)\left(t-t_{m}\right)^{k}
$$

which is the $(N+I)^{\text {th }}$ order approximate solution of Equation (10) obtained by DTM and thus the amplification factor of $\mathrm{E}_{\mathrm{I}}-\mathrm{MsDTM}$ with $N^{\text {th }}$ order and $I$ iterations is given by:

$$
R_{N, I}(z)=\sum_{k=0}^{N+I} \frac{z^{k}}{k !}
$$

which is the $(N+I)^{\text {th }}$ order Taylor expansion of $e^{z}$ and thus the $\mathrm{E}_{\mathrm{I}}-\mathrm{MsDTM}$ is not A-stable method.

From the stability analysis of MsDTM and EMsDTM, we get the following theorem.

\section{Theorem 8}

Enhanced-MsDTM with order $N$ and iterations $I$ and the MsDTM with order $(N+I)$ have the same stability region.

Also, we observe that applying the above procedure from Equation (21) to (26) on Equation (10) in a vector form results in the following theorem.

\section{Theorem 9}

Enhanced-MsDTM with order $N$ and iterations $I$ is equivalent to the MSDTM with order $(N+I)$ for the homogenous autonomous ODE system:

$$
\frac{d y}{d t}=A \boldsymbol{y}(t), \boldsymbol{y}\left(t_{0}\right)=\boldsymbol{y}_{0}
$$

where, $A$ is an ( $n \times x)$ constant matrix and $\mathbf{y}$ is an $(n \times 1)$ solution vector.

The stability regions of MsDTM and $\mathrm{E}_{\mathrm{I}}-\mathrm{MsDTM}$ at different orders $(N)$ and number of iterations $(I)$, respectively, are shown in Fig. 1.

\section{MsDTM-Padé Approximants}

The goal of Padé approximants is to make the maximum error of MsDTM, Equation (8), as small as possible by maintaining the accuracy far outside the radius of convergence of the series solution (Ehle, 1973; Rashidi and Mohimanian Pour, 2010a; 2010b; Lu, 2012; Kanth and Aruna, 2013; Domairry and Hatami, 2014) and to improve the stability property of the solution. Let the Padé approximation of the MsDTM series solution $S_{m}(t)$ on $\left[t_{m}, t_{m+l}\right]$ is the quotient of two polynomials $p_{m}(t)$ and $q_{m}(t)$ of order $p$ and $q$ respectively and $p+q=N$ (Ehle, 1973). We use the notation $S_{m, p a}(t)$ to denote the quotient:

$$
S_{m, P a}(t)=\frac{p_{m}(t)}{q_{m}(t)}, \text { fort } \in\left[t_{m}, t_{m+1}\right], m=0,1, \ldots \ldots, M-1
$$

Let the polynomials used in (28) are:

$$
\begin{aligned}
& p_{m}(t)=\sum_{k=0}^{p} P(k)\left(t-t_{m}\right)^{k}, \\
& q_{m}(t)=\sum_{k=0}^{q} Q(k)\left(t-t_{m}\right)^{k}, \text { with } Q(0)=1
\end{aligned}
$$




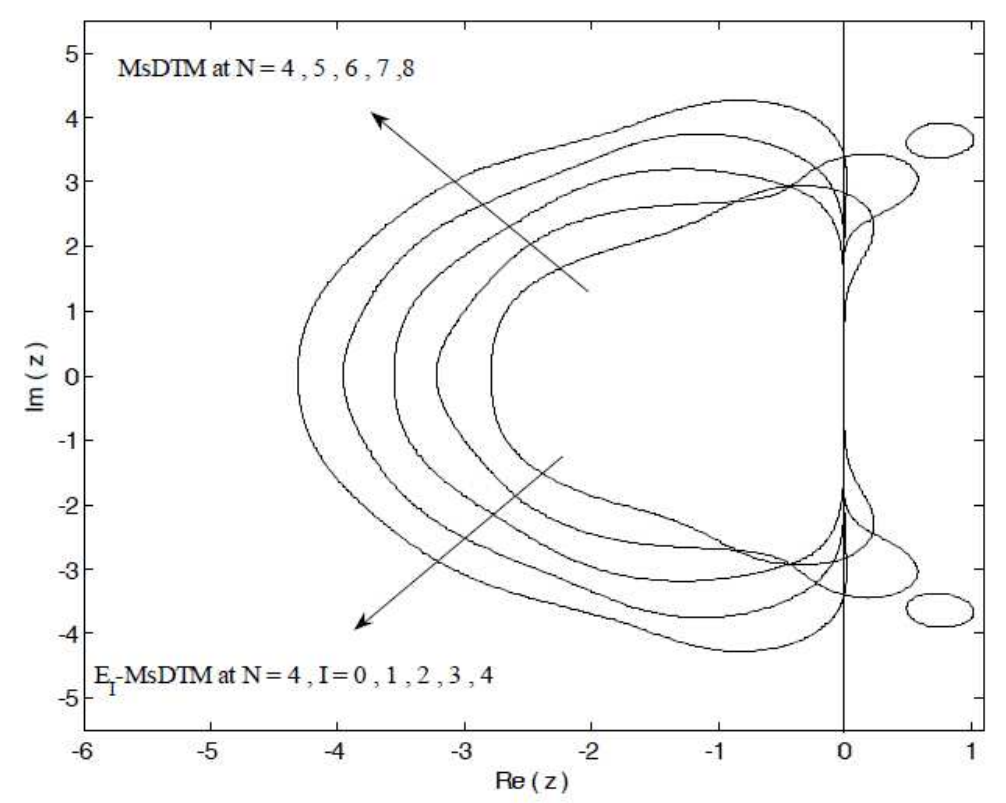

Fig. 1. Stability regions of MsDTM and $\mathrm{E}_{\mathrm{I}}-\mathrm{MsDTM}$ at different orders $(\mathrm{N})$ and number of iterations (I), respectively

The polynomials in (28) are constructed so that $S_{m}(t)$ and $S_{m, p a}(t)$ agree at $t=t_{m}$ and their derivatives up to $N$. Since $S_{m}(t)$ has a Taylor expansion defined in Equation (6), the formal power series:

$$
S_{m}(t)-S_{m, P a}(t)=S_{m}(t)-\frac{p_{m}(t)}{q_{m}(t)}=O\left(\left(t-t_{m}\right)^{N+1}\right)
$$

Determines the coefficients of $p_{m}(t)$ and $q_{m}(t)$. Multiplying (30) by $q_{m}(t)$ gives:

$$
\begin{aligned}
& \left(\sum_{k=0}^{N} Y(k)\left(t-t_{m}\right)^{k}\right)\left(\sum_{k=0}^{q} Q(k)\left(t-t_{m}\right)^{k}\right) \\
& -\left(\sum_{k=0}^{p} P(k)\left(t-t_{m}\right)^{k}\right)=\sum_{k=N+1}^{q N} C(k)\left(t-t_{m}\right)^{k}
\end{aligned}
$$

When the left side of (31) multiplied out and the coefficients of the powers of $\left(t-t_{m}\right)^{\mathrm{k}}$ are set equal to zero for $k=0,1, \ldots, N$, the result is a system of $N+1$ linear equations in the $N+1$ unknown coefficients of $p_{m}(t)$ and $q_{m}(t)$. Solving this linear system we get $S_{m, p a}(t)$ rational approximation of $S_{m}(t)$. From the local truncation error (30), it is clear that, for a fixed order $N$, as $\left(t-t_{m}\right) \rightarrow 0$, $S_{m, p a}(t) \rightarrow S_{m}(t)$ and thus the method is consistent.

\section{Stability Analysis of MsDTM-P}

For the stability of MsDTM with Padé approximants, it is well known that the Padé approximants of order $(p, q)$ are A-stable if and only if $2 \geq p-q \geq 0$ (Ehle, 1973; Hairer and Wanner, 1996,
Theorem 4.12), that is, the main diagonal and two sub diagonals in Padé approximants are A-stable. The absolute stability of MsDTM with diagonal and two sub diagonals Padé approximants is shown in Fig. 2.

\section{Numerical Experiments}

To demonstrate our results, a comparison of the accuracy, stability regions and computational efficiency of the MsDTM, E-MsDTM and MsDTM-P is presented through solving some linear and nonlinear practical problems with known exact solutions arising in electrical and chemical engineering. All calculations are carried out by MAPLE 14 software in a PC with a Pentium 2 $\mathrm{GHz}$ and $512 \mathrm{MB}$ of RAM.

\section{Example 1}

Figure 3 is a schematic diagram of a separately excited D.C motor where $R$ the armature resistance; $L$ the armature inductance; $I$ the armature current; $v$ the terminal voltage of the motor; $e$ the back electromotive force; $T$ the load torque; $J$ the torque of inertia and $\dot{\theta}=\omega$ is the motor speed. Taking $\omega_{r}=\omega / \Omega, I_{r}=i R / K_{b} \Omega, u=v / K_{b} \Omega$,

$B=b R / K_{b}^{2}, T_{L}=T R / \Omega K_{b}^{2}, \varepsilon=T_{e} / T_{m}$ as dimensionless variables, where $K_{b}$ is the constant of the torque, $\Omega$ is a nominal operating point, $T_{e}=L / R$ is the armature circuit time constant, $T_{m}=J R / K_{b}^{2}$ the mechanical time constant and $\tau=t / T_{m}$ as the time variable, then the transient behavior of the D.C motor shown in Fig. 3 can be described by following stiff ODE system. 


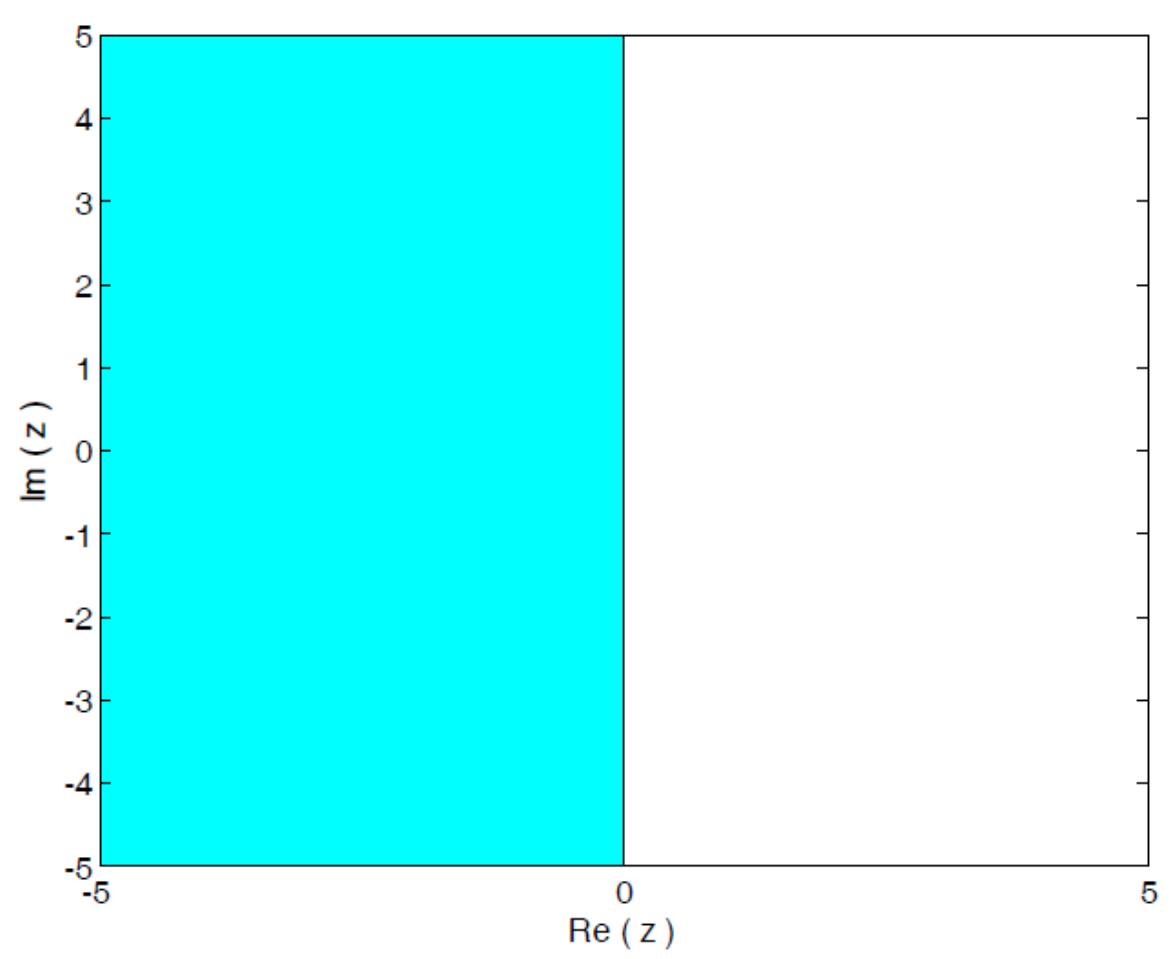

Fig. 2. Absolute stability of MsDTM with main and two sub diagonals Padé approximants

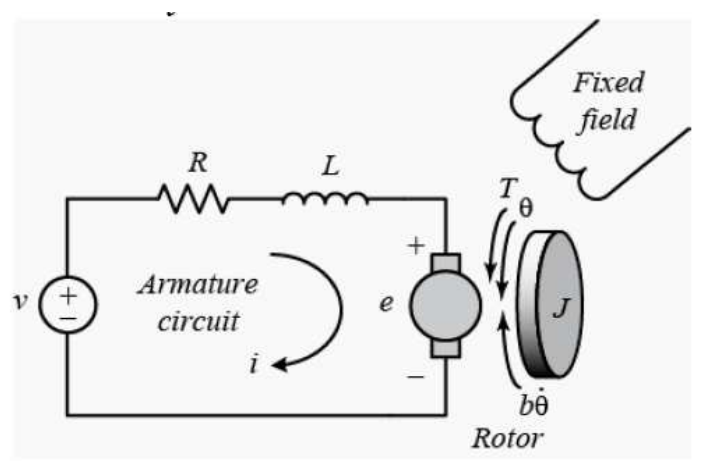

Fig. 3. A schematic diagram of a separately excited D.C motor

$$
\left.\begin{array}{l}
\frac{d \omega_{r}}{d \tau}=-B \omega_{r}+I_{r}-T_{L} \\
\varepsilon \frac{d I_{r}}{d \tau}=-\omega_{r}-I_{r}+u
\end{array}\right\}
$$

System (32) with parameters listed in Table 1 has been integrated using fourth order MsDTM (4MsDTM), enhanced fourth order MsDTM with one iteration ( $\left.\mathrm{E}_{1}-4 \mathrm{MsDTM}\right)$ and fourth order MsDTM with Padé approximant (4MsDTM-P) on the interval $[0,4]$ and the results are presented in figures and tables for different values of the step size $h$.

The errors have been defined as the maximum of the absolute pointwise errors for each component of the solution as:

$$
\begin{aligned}
& E_{\omega_{r}}=\left\|\omega_{r}\left(\tau_{j}\right)-\left(\omega_{r}\right)_{j}\right\|, \\
& E_{I_{r}}=\left\|I_{r}\left(\tau_{j}\right)-\left(I_{r}\right)_{j}\right\|, E_{\omega I}=\max \left\{E_{\omega_{r}}, E_{I_{r}}\right\}
\end{aligned}
$$

For system (32), at $\varepsilon=0.001$, the eigenvalues are $\lambda=-9.998 e+003,-1.2446 e+00$, the stiffness ratio is 802.625 and the drawback of MsDTM and EMsDTM are more severe, where for (32), the 4MsDTM is stable only if $h \leq h_{s 4},\left(h_{s 4}=2.7881 \mathrm{e}-003\right)$ and the $\mathrm{E}_{1}-4 \mathrm{MsDTM}$ is stable only if $h \leq h_{s 5}$, $\left(h_{s 5}=3.2202 \mathrm{e}-003\right)$.

Figure 4-6 show the stable and unstable solutions of (32) obtained by using 4MsDTM, $\mathrm{E}_{1}-4 \mathrm{MsDTM}$ and 4MsDTM-P at different values of time-step size $h$. In each figure, we choose the time step and the plotting range so that the differences between the obtained solutions are clear.

Figure 4 shows that the 4MsDTM solutions obtained at $\mathrm{h}=0.0025,0.0027\left(\mathrm{~h}<\mathrm{h}_{\mathrm{s} 4}\right)$, converge to the true solutions, while the solution obtained at $\mathrm{h}=$ $0.0028\left(\mathrm{~h}>\mathrm{h}_{\mathrm{s} 4}\right)$, is unstable and diverges far from the true solution. To overcome the stability restriction on using 4MsDTM with $\mathrm{h}=0.0028$ for (32), we should use smaller time-step size or higher order MsDTM, where the 5MsDTM has a larger stability region $\left(\mathrm{h}_{\mathrm{s} 5}=3.2202 \mathrm{e}-003\right)$ than 4 MsDTM. 


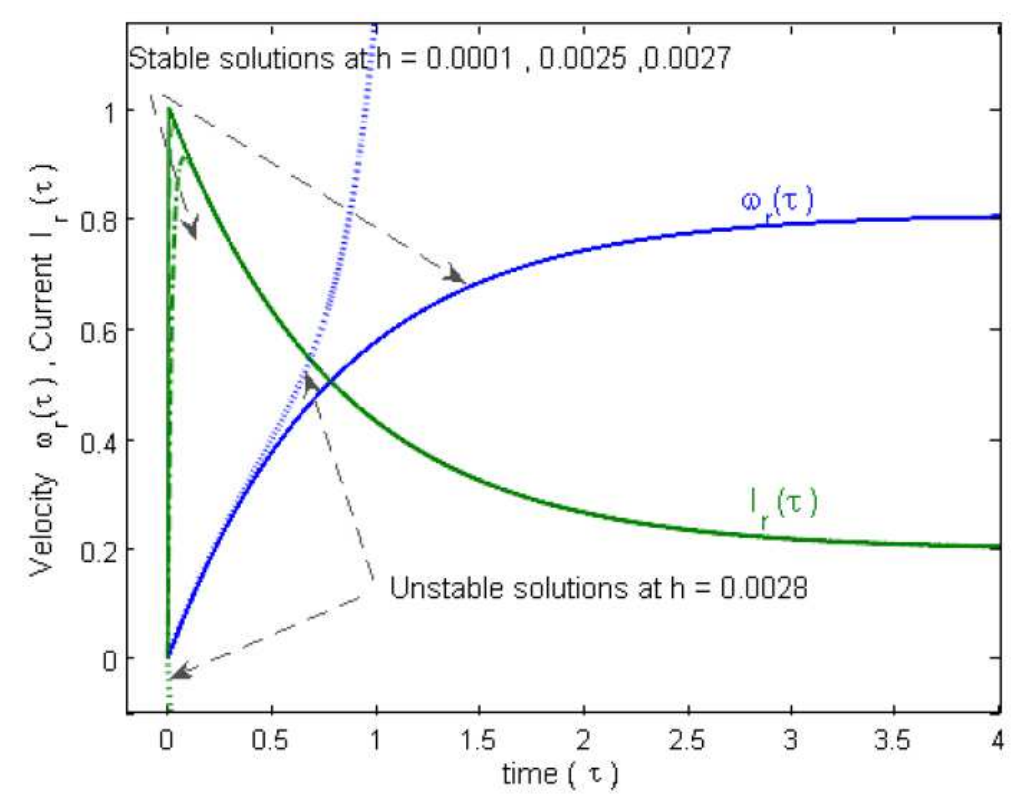

Fig. 4. 4MsDTM solutions for system (32) at different values of time-step size $\mathrm{h}$

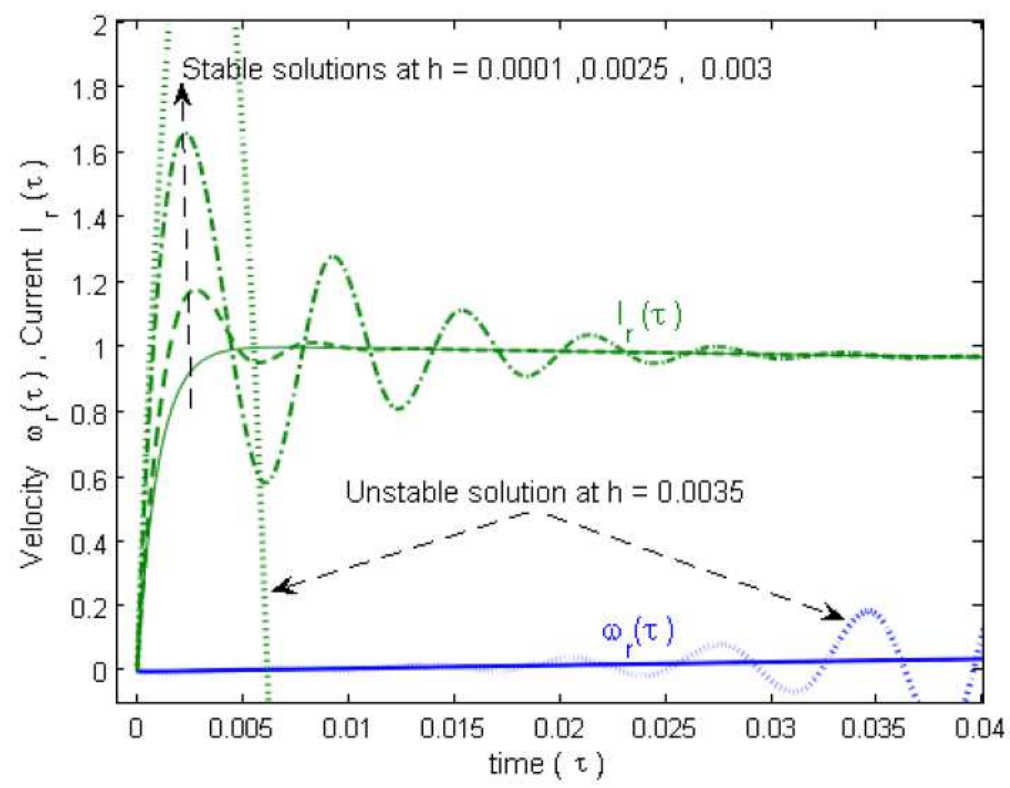

Fig. 5. $\mathrm{E}_{1}-4 \mathrm{MsDTM}$ solutions for system (32) at different values of time-step size $\mathrm{h}$

As shown in Fig. 5, although the $E_{1}-4 M s D T M$ solutions, at $\mathrm{h} 0.0025,0.003\left(\mathrm{~h}<\mathrm{h}_{\mathrm{s} 5}\right)$, exhibiting spurious oscillations at the layer region (Hairer and Wanner, 1996; Shyy et al., 1992; Yost and Rao, 2000), they remain bounded and decrease with time and thus the solutions are stable. At $\mathrm{h}=0.0035\left(\mathrm{~h}>\mathrm{h}_{\mathrm{s} 5}\right)$, the $\mathrm{E}_{1}-4 \mathrm{MsDTM}$ solutions oscillate wildly and quickly exit the range of the graph and therefore, there is a need to increase the order of the method or the number of iterations $I$, where $E_{1}$ $5 \mathrm{MsDTM}$ or $\mathrm{E}_{2}-4 \mathrm{MsDTM}$ has a larger stability region $\left(\mathrm{h}_{\mathrm{s} 6}=3.557 \mathrm{e}-003\right)$ than $\mathrm{E}_{1}-4 \mathrm{MsDTM}$.
Figure 6 shows that the 4MsDTM-P solutions are stables and converge to the true solutions even for larger time-step size.

Results in Table 2, show us that the 4MsDTM and $\mathrm{E}_{1}-4 \mathrm{MsDTM} \quad$ solutions at $h \geq 0.003$ and $h \geq 0.004$ respectively, do not approximate the true solutions of (32) correctly. We insert dashes (-) to indicate this phenomenon. Obviously, these step sizes are not enough to meet the stability restrictions where 4MsDTM and $\mathrm{E}_{1}-4 \mathrm{MsDTM}$ are not A-stable methods. 


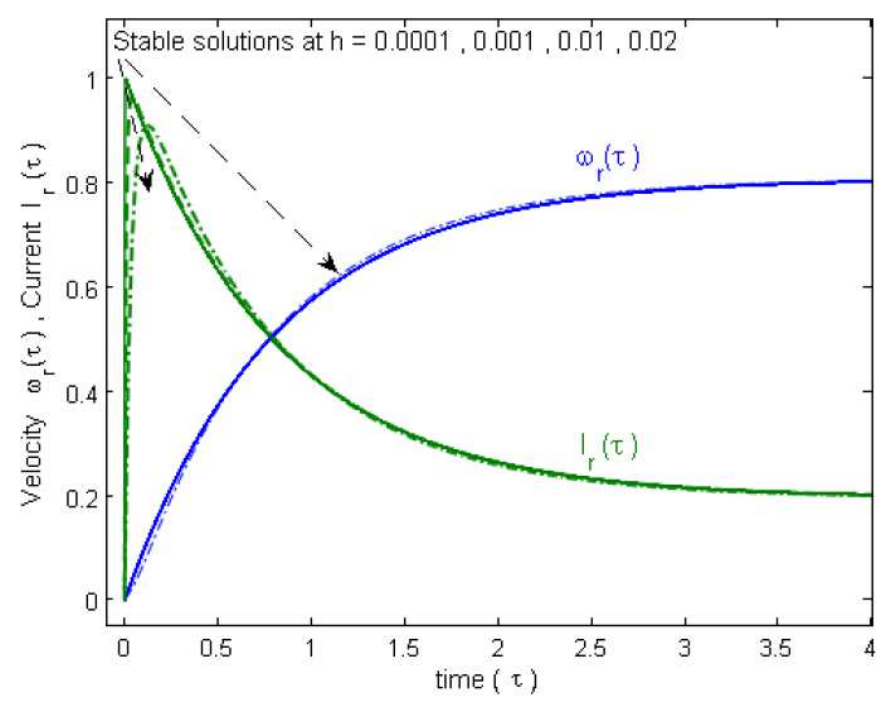

Fig. 6. 4MsDTM-P solutions for system (32) at different values of time-step size $\mathrm{h}$

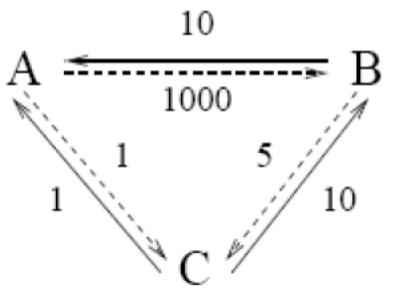

Fig. 7. A circular reaction

and therefore, there is a need to decrease the time-step to obtain accurate solutions, while the A-stable 4MsDTM-P performs better even for large time step.

Results in Table 2 also show us that the $\mathrm{E}_{1}-4 \mathrm{MsDTM}$ solutions are accurate better than the 4MsDTM solutions, while the 4MsDTM-P solutions are more accurate than $\mathrm{E}_{1}-4 \mathrm{MsDTM}$ solutions

Table 3 and 4 present a comparison of the maximum absolute error $E_{\omega I}$ and the CPU used time, respectively, for solving (32) using the $N \mathrm{MsDTM}, \mathrm{E}_{\mathrm{I}}-4 \mathrm{MsDTM}$ and $N$ MsDTM-P at different orders, $N$, number of iterations, $I$ and time-step size, $h$.

As shown from Table 3, the results of $(\mathrm{I}+4)$ MsDTM and $\mathrm{E}_{\mathrm{I}}-4 \mathrm{MsDTM}$ have the same accuracy and stability results, which confirms theoretical results in (Theorems 8 , 9), while the results of (I+4) MsDTM-P are more accurate. In addition, for a fixed order in NMsDTM or a fixed number of iterations in $\mathrm{E}_{\mathrm{I}}-4 \mathrm{MsDTM}$, as the step size decreases the numerical solution converges to the exact one. While, for a fixed step size $h$, the convergence of (NMsDTM, $\left.\mathrm{E}_{\mathrm{I}}-4 \mathrm{MsDTM}\right)$ only begins when $(N, \mathrm{I})$ are $(O(K h), O(L h))$, respectively, where for (32), $K=L=O\left(\varepsilon^{-1}\right)$ ([Vulanovic, 1989, Theorem 1]; (El-Zahar and EL-Kabeir, 2013, Lemma 3.2); Zhao and Xiao, 2010; Zhao et al., 2014).
Results in Table 4, show us that, for the same step size $h$, the $(\mathrm{I}+4)$ MsDTM has the lowest CPU usage and the $\mathrm{E}_{\mathrm{I}}-4 \mathrm{MsDTM}$ has the highest CPU usage in solving (32). Moreover, increasing the order $N$ of $N$ MsDTM is computationally cheaper than increasing the order of $N$ MsDTM-P, while increasing the number of iterations $I$ in $\mathrm{E}_{\mathrm{I}}-4 \mathrm{MsDTM}$ is computationally more expensive, where for $I>1$ successive analytical integrations are required (I-1)-times and therefore the MsDTM is the lowest computational cost while the E-MsDTM is the highest computational cost.

\section{Example 2}

Figure 7 describes a circular reaction with 3 substances $A, B$ and $C$, with initial values $A(0)=1, B(0)$ $=2$ and $C(0)=3$. The system of ODEs describing the circular reaction in Fig. 7 is as follows:

$$
\frac{d}{d t}\left[\begin{array}{l}
A(t) \\
B(t) \\
C(t)
\end{array}\right]=\left[\begin{array}{ccc}
-1001 & 10 & 1 \\
1000 & -15 & 10 \\
1 & 5 & -11
\end{array}\right]\left[\begin{array}{l}
A(t) \\
B(t) \\
C(t)
\end{array}\right]
$$

For system (33), the eigenvalues are $\lambda=-1.0110 e 03,-1.5964 e 01,0.00$ and therefore the stiffness ratio is 63.33 and we have $\left(h_{s 4}=2.7550 \mathrm{e}-003, h_{s 5}=3.1820 \mathrm{e}-003\right)$. The system has been integrated on the interval $[0,1]$ and the numerical results are presented in Fig. 8-10 and Table 5 and 6 using MsDTM, E-MsDTM and MsDTM-P at different orders, $N$, number of iterations, $I$ and timestep size $h$. The present numerical results for system (33) provide support for the earlier analysis of the results presented for system (32), which confirm our theoretical results. 


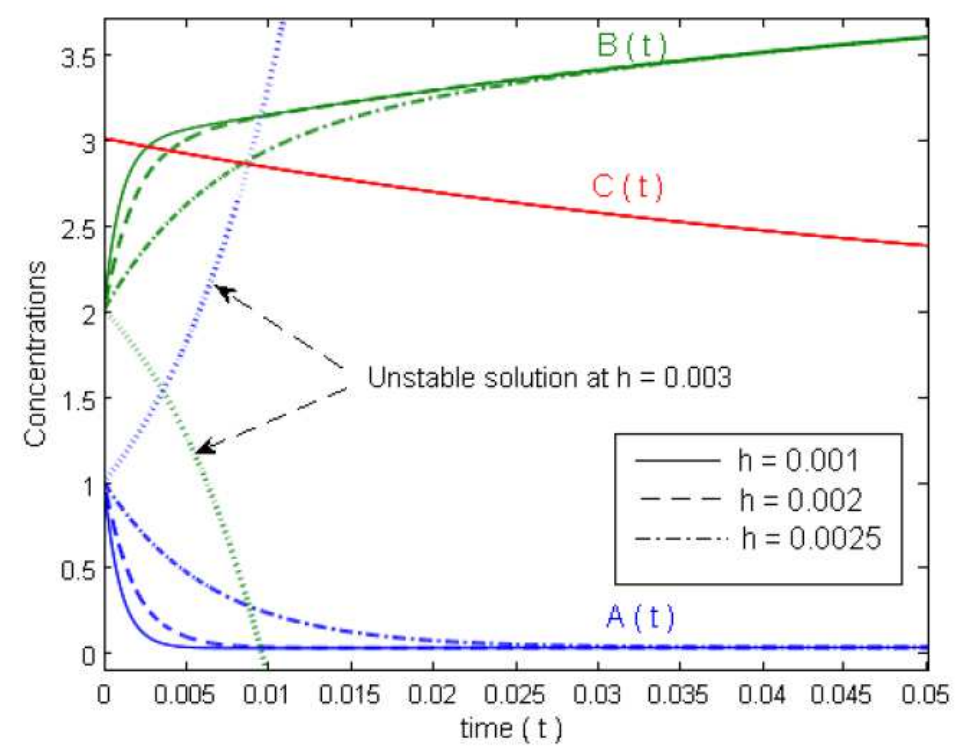

Fig. 8. 4MsDTM solutions for the system (33) at different values of time-step size $\mathrm{h}$

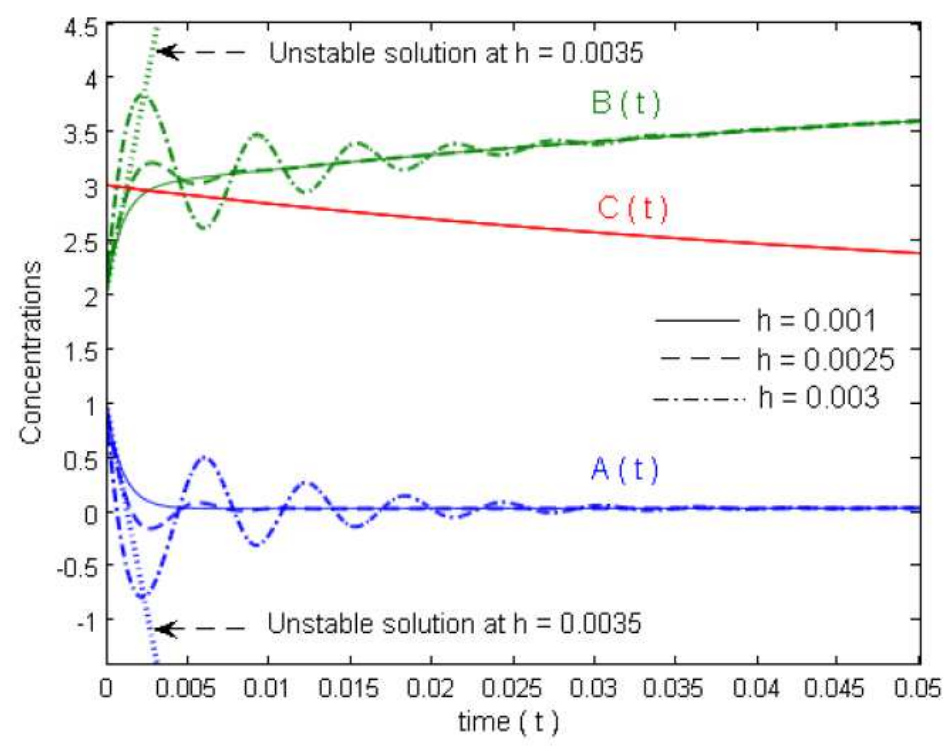

Fig. 9. E1-4MsDTM solutions for the system (33) at different values of time-step size h

\section{Example 3}

Consider the nonlinear system of ODEs ( $\mathrm{Wu}$ and Xia, 2001):

$$
Y^{\prime}=-B Y+U W
$$

where:

$$
Y(0)=\left(\begin{array}{l}
-1 \\
-1 \\
-1 \\
-1
\end{array}\right), U=\frac{1}{2}\left(\begin{array}{rrrr}
-1 & 1 & 1 & 1 \\
1 & -1 & 1 & 1 \\
1 & 1 & -1 & 1 \\
1 & 1 & 1 & -1
\end{array}\right), B=U\left(\begin{array}{llll}
d_{1} & & & \\
& d_{2} & & \\
& & d_{3} & \\
& & & d_{4}
\end{array}\right) U
$$

$$
W=\left(\begin{array}{l}
z_{1}^{2} \\
z_{2}^{2} \\
z_{3}^{2} \\
z_{4}^{2}
\end{array}\right), Z=\left(\begin{array}{l}
z_{1} \\
z_{2} \\
z_{3} \\
z_{4}
\end{array}\right)=U Y, D=\left(\begin{array}{l}
d_{1} \\
d_{2} \\
d_{3} \\
d_{4}
\end{array}\right)=\left(\begin{array}{c}
1000 \\
800 \\
-10 \\
0.001
\end{array}\right)
$$

The exact solution of (34) is:

$$
Y=U Z
$$

where: 


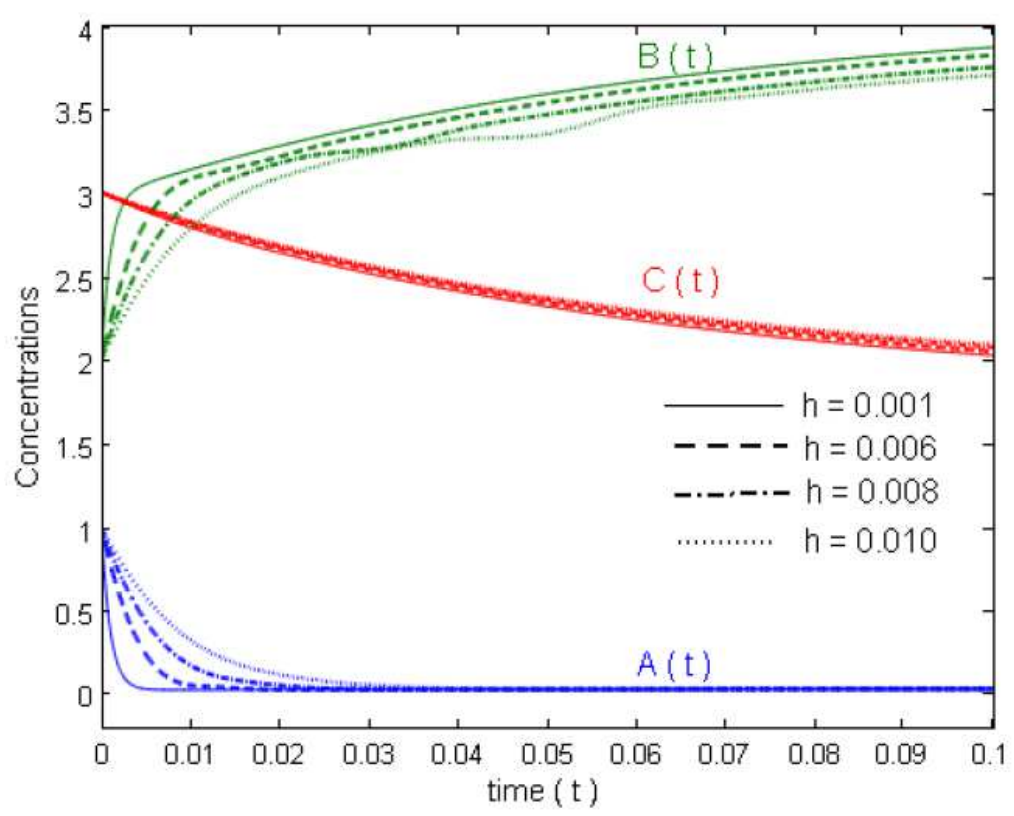

Fig. 10. 4MsDTM-P stable solutions for the system (33) at different values of time-step size $h$

Table 1. Parameters of the separately excited D.C motor

\begin{tabular}{ll}
\hline $\mathrm{J}=8 \times 10^{-3} \mathrm{~kg} \cdot \mathrm{m} 2$ & $\mathrm{~K}_{\mathrm{b}}=0.043 \mathrm{~V} / \mathrm{rad} / \mathrm{s}$ \\
$\mathrm{R}=1.5 \Omega$ & $\Omega=279.07 \mathrm{rad} / \mathrm{s}$ \\
$\mathrm{b}=3 \times 10^{-4} \mathrm{~N} \cdot \mathrm{m} / \mathrm{rad} / \mathrm{s}$ & $\mathrm{V}_{\mathrm{a}}=12 \mathrm{~V}$ \\
$\mathrm{~L}=9.735 \mathrm{mH}$ & $\mathrm{T}_{\mathrm{L}}=0$ \\
\hline
\end{tabular}

Table 2. Maximum absolute errors of 4MsDTM, $\mathrm{E}_{1}-4 \mathrm{MsDTM}$ and 4MsDTM-P at different time-step size h for system (32)

\begin{tabular}{lllllll}
\hline \multicolumn{5}{c}{$E_{\omega_{r}}$} \\
\hline$h$ & 4MsDTM & E1-4MsDTM & 4MsDTM-P & 4 4MsDTM & E1-4MsDTM & 4MsDTM-P \\
0.0200 & - & - & $2.7050 \mathrm{e}-2$ & - & - & $5.3853 \mathrm{e}-1$ \\
0.0100 & - & - & $4.0418 \mathrm{e}-3$ & - & - & $2.9919 \mathrm{e}-1$ \\
0.0050 & - & - & $4.6199 \mathrm{e}-4$ & - & - & $9.7038 \mathrm{e}-2$ \\
0.0040 & - & - & $2.2318 \mathrm{e}-4$ & - & - & $5.8205 \mathrm{e}-2$ \\
0.0030 & - & $6.9808 \mathrm{e}-4$ & $8.6548 \mathrm{e}-5$ & - & $6.9721 \mathrm{e}-1$ & $2.6954 \mathrm{e}-2$ \\
0.0027 & $8.1048 \mathrm{e}-4$ & $3.3816 \mathrm{e}-4$ & $6.0986 \mathrm{e}-5$ & $8.0947 \mathrm{e}-1$ & $3.8269 \mathrm{e}-1$ & $1.9728 \mathrm{e}-2$ \\
0.0025 & $5.6554 \mathrm{e}-4$ & $2.4684 \mathrm{e}-4$ & $4.7189 \mathrm{e}-5$ & $5.6483 \mathrm{e}-1$ & $2.4653 \mathrm{e}-1$ & $1.5554 \mathrm{e}-2$ \\
0.0020 & $1.9770 \mathrm{e}-4$ & $6.8496 \mathrm{e}-5$ & $2.2180 \mathrm{e}-5$ & $1.9746 \mathrm{e}-1$ & $6.8410 \mathrm{e}-2$ & $7.4707 \mathrm{e}-3$ \\
0.0010 & $1.1661 \mathrm{e}-6$ & $1.2096 \mathrm{e}-6$ & $1.9070 \mathrm{e}-6$ & $1.1647 \mathrm{e}-3$ & $1.2081 \mathrm{e}-3$ & $5.3772 \mathrm{e}-4$ \\
\hline
\end{tabular}

Table 3. Maximum absolute errors $\mathrm{E}_{\omega \mathrm{I}}$ of different methods at different orders and time-step size for system (32)

\begin{tabular}{llllll}
\hline$h$ & 0.004 & 0.003 & 0.0025 & 0.002 & 0.001 \\
\hline 5MsDTM & - & $6.9721 \mathrm{E}-1$ & $2.4653 \mathrm{E}-1$ & $6.8410 \mathrm{E}-2$ & $1.2081 \mathrm{E}-3$ \\
6MsDTM & - & $3.1124 \mathrm{E}-1$ & $9.1200 \mathrm{E}-2$ & $2.0123 \mathrm{E}-2$ & $1.7536 \mathrm{E}-4$ \\
7 MsDTM & - & $1.2052 \mathrm{E}-1$ & $2.9297 \mathrm{E}-2$ & $5.1465 \mathrm{E}-3$ & $2.2167 \mathrm{E}-5$ \\
8MsDTM & $5.0842 \mathrm{e}-1$ & $4.1227 \mathrm{E}-2$ & $8.3206 \mathrm{E}-3$ & $1.1647 \mathrm{E}-3$ & $2.4860 \mathrm{E}-6$ \\
5MsDTM-P [2/3] & $5.7084 \mathrm{E}-2$ & $2.6326 \mathrm{E}-2$ & $1.5139 \mathrm{E}-2$ & $7.2327 \mathrm{E}-3$ & $5.0646 \mathrm{E}-4$ \\
6MsDTM-P [3/3] & $5.3599 \mathrm{E}-3$ & $1.5185 \mathrm{E}-3$ & $6.3195 \mathrm{E}-3$ & $2.0093 \mathrm{E}-4$ & $3.8045 \mathrm{E}-6$ \\
7MsDTM-P [3/4] & $2.6364 \mathrm{E}-4$ & $4.2917 \mathrm{E}-5$ & $1.2348 \mathrm{E}-5$ & $4.0629 \mathrm{E}-6$ & $5.4844 \mathrm{E}-8$ \\
8MsDTM-P [4/4] & $2.9289 \mathrm{E}-4$ & $4.9229 \mathrm{E}-5$ & $1.4552 \mathrm{E}-5$ & $3.0193 \mathrm{E}-6$ & $1.4690 \mathrm{E}-8$ \\
$\mathrm{E}_{1}-4 \mathrm{MsDTM}$ & - & $6.9721 \mathrm{E}-1$ & $2.4653 \mathrm{E}-1$ & $6.8410 \mathrm{E}-2$ & $1.2081 \mathrm{E}-3$ \\
$\mathrm{E}_{2}-4 \mathrm{MsDTM}$ & - & $3.1124 \mathrm{E}-1$ & $9.1200 \mathrm{E}-2$ & $2.0123 \mathrm{E}-2$ & $1.7536 \mathrm{E}-4$ \\
$\mathrm{E}_{3}-4 \mathrm{MsDTM}$ & - & $1.2052 \mathrm{E}-1$ & $2.9297 \mathrm{E}-2$ & $5.1465 \mathrm{E}-3$ & $2.2167 \mathrm{E}-5$ \\
$\mathrm{E}_{4}-4 \mathrm{MsDTM}$ & $5.0842 \mathrm{e}-1$ & $4.1227 \mathrm{E}-2$ & $8.3206 \mathrm{E}-3$ & $1.1648 \mathrm{E}-3$ & $2.4860 \mathrm{E}-6$ \\
\hline
\end{tabular}


E.R. El-Zahar et al. / American Journal of Applied Sciences 2015, 12 (5): 304.320 DOI: 10.3844/ajassp.2015.304.320

Table 4. CPU time(s) in Sec used in solving system (32)

\begin{tabular}{llllll}
\hline$h$ & 0.004 & 0.003 & 0.0025 & 0.002 & 0.001 \\
\hline 5MsDTM & - & 00.641 & 00.750 & 00.953 & 01.922 \\
6MsDTM & - & 00.703 & 00.813 & 01.000 & 01.953 \\
7MsDTM & - & 00.813 & 00.891 & 01.047 & 02.141 \\
8MsDTM & 00.766 & 00.906 & 01.031 & 01.140 & 02.281 \\
5MsDTM-P [2/3] & 02.625 & 03.344 & 04.016 & 05.015 & 10.125 \\
6MsDTM-P [3/3] & 02.750 & 03.703 & 04.500 & 05.593 & 11.078 \\
7MsDTM-P [3/4] & 03.437 & 04.641 & 05.500 & 06.875 & 14.000 \\
8MsDTM-P [4/4] & 03.860 & 06.313 & 06.094 & 07.687 & 15.375 \\
E $_{1}-4$ MsDTM & - & 03.469 & 04.188 & 05.188 & 10.375 \\
E $_{2}-4$ MsDTM & - & 06.968 & 08.407 & 10.531 & 20.938 \\
E $_{3}-4$ MsDTM & - & 10.125 & 12.109 & 16.047 & 33.875 \\
E $_{4}-4$ MsDTM & 10.512 & 12.365 & 15.674 & 20.021 & 41.108 \\
\hline
\end{tabular}

Table 5. Maximum absolute errors of different methods at different orders and time-step size for system (33)

\begin{tabular}{llllll}
\hline$h$ & 0.004 & 0.003 & 0.0025 & 0.002 & 0.001 \\
\hline 5MsDTM & - & $7.2348 \mathrm{e}-1$ & $2.5594 \mathrm{e}-1$ & $7.1061 \mathrm{e}-2$ & $1.2565 \mathrm{e}-3$ \\
6MsDTM & - & $3.2698 \mathrm{e}-1$ & $9.5856 \mathrm{e}-2$ & $2.1161 \mathrm{e}-2$ & $1.8450 \mathrm{e}-4$ \\
7 MsDTM & - & $1.2818 \mathrm{e}-1$ & $3.1173 \mathrm{e}-2$ & $5.4785 \mathrm{e}-3$ & $2.3621 \mathrm{e}-5$ \\
8MsDTM & $5.4701 \mathrm{e}-1$ & $4.4388 \mathrm{e}-2$ & $8.9620 \mathrm{e}-3$ & $1.2550 \mathrm{e}-3$ & $2.6813 \mathrm{e}-6$ \\
5MsDTM-P [2/3] & $1.3698 \mathrm{e}-1$ & $3.7719 \mathrm{e}-2$ & $1.6635 \mathrm{e}-2$ & $6.0714 \mathrm{e}-3$ & $2.3814 \mathrm{e}-4$ \\
6MsDTM-P [3/3] & $5.3980 \mathrm{e}-3$ & $1.5400 \mathrm{e}-3$ & $6.4337 \mathrm{e}-4$ & $2.0542 \mathrm{e}-4$ & $3.9269 \mathrm{e}-6$ \\
7MsDTM-P [3/4] & $2.2854 \mathrm{e}-3$ & $5.5555 \mathrm{e}-4$ & $2.0054 \mathrm{e}-4$ & $5.4601 \mathrm{e}-5$ & $8.0908 \mathrm{e}-7$ \\
8MsDTM-P [4/4] & $3.4722 \mathrm{e}-4$ & $5.8611 \mathrm{e}-5$ & $1.7378 \mathrm{e}-5$ & $3.6186 \mathrm{e}-6$ & $3.2323 \mathrm{e}-7$ \\
$\mathrm{E}_{1}-4$ MsDTM & - & $7.2348 \mathrm{e}-1$ & $2.5594 \mathrm{e}-1$ & $7.1061 \mathrm{e}-2$ & $1.2565 \mathrm{e}-3$ \\
$\mathrm{E}_{2}-4$ MsDTM & - & $3.2698 \mathrm{e}-1$ & $9.5856 \mathrm{e}-2$ & $2.1161 \mathrm{e}-2$ & $1.8450 \mathrm{e}-4$ \\
$\mathrm{E}_{3}-4$ MsDTM & - & $1.2818 \mathrm{e}-1$ & $3.1173 \mathrm{e}-2$ & $5.4785 \mathrm{e}-3$ & $2.3621 \mathrm{e}-5$ \\
$\mathrm{E}_{4}-4$ MsDTM & $5.4701 \mathrm{e}-1$ & $4.4388 \mathrm{e}-2$ & $8.9620 \mathrm{e}-3$ & $1.2551 \mathrm{e}-3$ & $2.6813 \mathrm{e}-6$ \\
\hline
\end{tabular}

Table 6. CPU time(s) in Sec used in solving system (33)

\begin{tabular}{llllll}
\hline$h$ & 0.004 & 0.003 & 0.0025 & 0.002 & 0.001 \\
\hline 5MsDTM & - & 00.312 & 00.360 & 00.422 & 00.906 \\
6MsDTM & - & 00.359 & 00.359 & 00.500 & 01.031 \\
7MsDTM & - & 00.401 & 00.417 & 00.513 & 01.094 \\
8MsDTM & 00.312 & 00.406 & 00.469 & 00.578 & 01.156 \\
5MsDTM-P [2/3] & 01.015 & 01.266 & 01.500 & 01.922 & 03.879 \\
6MsDTM-P [3/3] & 01.094 & 01.407 & 01.656 & 02.156 & 04.188 \\
7MsDTM-P [3/4] & 01.469 & 01.766 & 02.156 & 02.672 & 05.234 \\
8MsDTM-P [4/4] & 01.484 & 02.031 & 02.250 & 02.859 & 05.781 \\
E $_{1}$-4MsDTM & - & 01.375 & 01.594 & 01.938 & 03.875 \\
E $_{2}-4$ MsDTM & - & 02.625 & 03.047 & 03.828 & 07.734 \\
E $_{3}$-MsDTM & - & 12.031 & 09.906 & 12.282 & 18.140 \\
E $_{4}-4$ MsDTM & 19.325 & 24.841 & 29.898 & 35.128 & 48.125 \\
\hline
\end{tabular}

Table 7. Maximum absolute errors of 4MsDTM, $\mathrm{E}_{1}-4 \mathrm{MsDTM}$ and 4MsDTM-P for system (34)

\begin{tabular}{llll}
\hline$h$ & 4 MsDTM & E1-4MsDTM & $4 M s D T M-P$ \\
\hline 0.0050 & - & - & $7.5894 \mathrm{e}-2$ \\
0.0040 & - & - & $4.7552 \mathrm{e}-2$ \\
0.0030 & - & $4.5933 \mathrm{e}-1$ & $1.9585 \mathrm{e}-2$ \\
0.0027 & $5.6182 \mathrm{e}-1$ & $2.5191 \mathrm{e}-1$ & $1.4148 \mathrm{e}-2$ \\
0.0025 & $3.9178 \mathrm{e}-1$ & $1.6220 \mathrm{e}-1$ & $1.1054 \mathrm{e}-2$ \\
0.0020 & $1.3673 \mathrm{e}-1$ & $4.4960 \mathrm{e}-2$ & $5.1837 \mathrm{e}-3$ \\
0.0010 & $4.8999 \mathrm{e}-3$ & $7.9266 \mathrm{e}-4$ & $3.5344 \mathrm{e}-4$ \\
\hline
\end{tabular}


Table 8. Maximum absolute errors of different methods at different orders for system (34)

\begin{tabular}{|c|c|c|c|c|}
\hline$h$ & 0.004 & 0.003 & 0.002 & 0.001 \\
\hline 5MsDTM & - & $4.7058 \mathrm{e}-1$ & $4.6153 \mathrm{e}-2$ & $8.1526 \mathrm{e}-4$ \\
\hline 6MsDTM & - & $2.1115 \mathrm{e}-1$ & $1.3697 \mathrm{e}-2$ & $1.1990 \mathrm{e}-4$ \\
\hline 7MsDTM & $8.9639 \mathrm{e}-1$ & $8.6648 \mathrm{e}-2$ & $3.7323 \mathrm{e}-3$ & $1.6268 \mathrm{e}-5$ \\
\hline 8MsDTM & $4.1510 \mathrm{e}-1$ & $3.4088 \mathrm{e}-2$ & $9.7866 \mathrm{e}-4$ & $2.1338 \mathrm{e}-6$ \\
\hline 5MsDTM-P [2/3] & $4.2018 \mathrm{e}-2$ & $1.2326 \mathrm{e}-2$ & $1.9659 \mathrm{e}-3$ & $9.1152 \mathrm{e}-5$ \\
\hline 6MsDTM-P [3/3] & $3.4206 \mathrm{e}-3$ & $1.0154 \mathrm{e}-3$ & $1.4181 \mathrm{e}-4$ & $5.5071 \mathrm{e}-5$ \\
\hline 7MsDTM-P [3/4] & $3.7350 \mathrm{e}-3$ & $6.7155 \mathrm{e}-4$ & $5.1114 \mathrm{e}-5$ & $6.9606 \mathrm{e}-7$ \\
\hline 8MsDTM-P[4/4] & $2.2286 \mathrm{e}-4$ & $3.8427 \mathrm{e}-5$ & $2.4243 \mathrm{e}-6$ & $5.6783 \mathrm{e}-8$ \\
\hline $\mathrm{E}_{1}-4 \mathrm{MsDTM}$ & - & $4.5933 \mathrm{e}-1$ & $4.4960 \mathrm{e}-2$ & $7.9266 \mathrm{e}-4$ \\
\hline $\mathrm{E}_{2}-4 \mathrm{MsDTM}$ & - & $1.9605 \mathrm{e}-1$ & $1.2665 \mathrm{e}-2$ & $1.1029 \mathrm{e}-4$ \\
\hline $\mathrm{E}_{3}-4 \mathrm{MsDTM}$ & $6.7896 \mathrm{e}-1$ & $7.3224 \mathrm{e}-2$ & $3.1266 \mathrm{e}-3$ & $1.3482 \mathrm{e}-5$ \\
\hline $\mathrm{E}_{4}-4 \mathrm{MsDTM}$ & $2.9982 \mathrm{e}-1$ & $2.4318 \mathrm{e}-2$ & $6.8748 \mathrm{e}-4$ & $1.4706 \mathrm{e}-6$ \\
\hline
\end{tabular}

Table 9. CPU time(s) in Sec used in solving system (34)

\begin{tabular}{lllll}
\hline$h$ & 0.004 & 0.003 & 0.002 & 0.001 \\
\hline 5MsDTM & - & 08.181 & 12.219 & 24.547 \\
6MsDTM & - & 09.859 & 14.703 & 29.579 \\
7MsDTM & 08.750 & 11.656 & 17.422 & 34.813 \\
8MsDTM & 10.125 & 13.516 & 20.156 & 40.406 \\
5MsDTM-P [2/3] & 07.109 & 09.532 & 14.235 & 28.594 \\
6MsDTM-P [3/3] & 08.516 & 11.359 & 16.984 & 34.047 \\
7MsDTM-P [3/4] & 10.140 & 13.547 & 20.344 & 40.719 \\
8MsDTM-P[4/4] & 11.641 & 15.672 & 23.359 & 46.875 \\
E $_{1}$-4MsDTM & - & 10.141 & 15.156 & 30.219 \\
E $_{2}$-MsDTM & - & 14.215 & 21.894 & 48.716 \\
E $_{3}$-4MsDTM & 12.014 & 35.097 & 43.810 & 65.125 \\
E $_{4}$-4MsDTM & 28.451 & 44.384 & 55.127 & 78.124 \\
\hline
\end{tabular}

$$
Y=\left(\begin{array}{l}
y_{1 E}(t) \\
y_{2 E}(t) \\
y_{3 E}(t) \\
y_{4 E}(t)
\end{array}\right), Z=\left(\begin{array}{l}
z_{1}(t) \\
z_{2}(t) \\
z_{3}(t) \\
z_{4}(t)
\end{array}\right), z_{i}(t)=\frac{d_{i}}{1-\left(1+\mathrm{d}_{i}\right) e^{d_{i} t}}, i=1,2,3,4
$$

The problem has been integrated on the interval $[0,1]$ and the numerical results are presented in Table 7-9 using MsDTM, E-MsDTM and MsDTM-P at different orders, $N$, number of iterations, $I$ and time-step size $h$.

Results in Table 7 show that, the $\mathrm{E}_{1}-4 \mathrm{MsDTM}$ solutions are accurate better than the 4MsDTM solutions, while the 4MsDTM-P solutions are more accurate and have a good stability property.

Results in Table 8 show that, the $\mathrm{E}_{\mathrm{I}}-4 \mathrm{MsDTM}$ results are little better than the $(\mathrm{I}+4) \mathrm{MsDTM}$ results, while the $(\mathrm{I}+4)$ MsDTM-P results are more accurate than the $(\mathrm{I}+4)$ MsDTM and $\mathrm{E}_{\mathrm{I}}-4 \mathrm{MsDTM}$ results.

Results in Table 4, 6, 9 show us that, the CPU usage in solving (34) is extremely higher than CPU usage in (32) and (33) and thus is due to the nonlinearity of (34). But we still have MsDTM with the lowest computational cost and E-MsDTM with the highest computational cost.

\section{Conclusion}

In this study, we have presented an effective comparison among three explicit one step semianalytical numerical methods for solving stiff ODE systems. The methods are the MsDTM, E-MsDTM and MsDTM-P. The error and stability analysis of each method is presented. The error analysis shows that the methods are consistent and so they closely match the given IVP (1) when the step size $h$ is sufficiently small. The stability analysis shows that the $\mathrm{E}_{\mathrm{T}}-N \mathrm{MsDTM}$ have stability regions larger than those with $N$ MsDTM and both of them are conditionally stable methods, while NMsDTM-P is A-stable method and larger time steps can be used hence suitable for the stiff ODE systems. In addition, the $(N+\mathrm{I}) \mathrm{MsDTM}$ and $\mathrm{E}_{\mathrm{I}}-N \mathrm{MsDTM}$ have the same stability regions (Theorem 8 ) and are equivalent for linear homogenous autonomous initial-value ODE system (Theorem 9). Also, the present analysis of the $\mathrm{E}_{\mathrm{I}}-N \mathrm{MsDTM}$ shows that it is equivalent to the multiplestep Picard iteration method with NDTM solution as the zeroth approximation of $y_{m}(t)$. We have applied the methods on three linear and nonlinear practical problems with known exact solutions and the numerical results are presented in figures and tables for comparison. The numerical results confirm our theoretical ones and additionally show that:

- As the step size decreases or the order increases or the number of iterations for E-MsDTM increases, the accuracy of the methods increases

- For the same order and step size, the E-MsDTM solutions are accurate better than the MsDTM 
solutions, while the MsDTM-P solutions are more accurate than E-MsDTM solutions

- For the same step size, the $\mathrm{E}_{\mathrm{I}}-N \mathrm{MsDTM}$ and $(N+\mathrm{I}) \mathrm{MsDTM}$ have the same results for linear homogenous autonomous ODE systems, while for nonlinear ODE systems the $\mathrm{E}_{\mathrm{I}}-N \mathrm{MsDTM}$ solutions are accurate little better than the $(N+\mathrm{I}) \mathrm{MsDTM}$ solutions

- $\quad$ Numerically, the convergence of $\mathrm{E}_{I}$-MsDTM begins when $I$ is of order $O(L h)$, while begins for MsDTM when $N$ is of order $O(K h)$

From the view of computations, increasing the order of the MsDTM or MsDTM-P is computationally cheaper than increasing the number of iterations in E-MsDTM.

We conclude that the MsDTM-P is more suitable than classical MsDTM and E-MsDTM for solving stiff ODE systems because of its stability, higher accuracy and a relatively low computational cost.

\section{Acknowledgement}

This project was supported by the Deanship of Scientific Research at Salman bin Abdulaziz University under the research project \# 2014/01/1434.

\section{Author's Contributions}

E.R. El-Zahar: Participated in overall error and stability analysis, solving stiff systems, analysis and interpretation of the results, comparisons among algorithms, edited the manuscript.

H.M. Habib: Participated in error and stability analysis of MsDTM, solving stiff electrical system, analysis and interpretation of the results, critical revision, contributed to the writing of the manuscript.

M.M. Rashidi: Participated in error and stability analysis of E-MsDTM, solving stiff reaction system, analysis and interpretation of the results, critical revision, contributed to the writing of the manuscript.

I.M. El-Desoky: Participated in error and stability analysis of MsDTM-P, solving stiff nonlinear system, analysis and interpretation of the results, critical revision, contributed to the writing of the manuscript.

\section{Ethics}

The authors confirm that this work is original and has not been published elsewere.

\section{References}

Hassan, A.H.I.H., 2008. Application to differential transformation method for solving systems of differential equations. Applied Math. Modell., 32: 2552-2559. DOI: 10.1016/j.apm.2007.09.025
Adomian, G., R. Rach and M. Elrod, 1988. The decomposition method applied to stiff systems. Math. Comput. Simulat., 30: 271-276. DOI: 10.1016/0378-4754(88)90006-7

Ahmad, R.R., N. Yaacob and A.H.M. Murid, 2004. Explicit methods in solving stiff ordinary differential equations. Int. J. Comput. Math., 81: 1407-1415. DOI: $10.1080 / 00207160410001661744$

Ahmad, R.R. and N. Yaacob, 2005. Third-order composite Runge-Kutta method for stiff problems. Int. J. Comput. Math., 82: 1221-1226. DOI: $10.1080 / 00207160512331331039$

Al-Amr, M.O., 2014. New applications of reduced differential transform method. Alexandria Eng. J., 53: 243-247. DOI: 10.1016/j.aej.2014.01.003

Alomari, A.K., M.S.M. Noorani and R. Nazar, 2010. On a new reliable algorithm. Int. J. Differential Equ. DOI: $10.1155 / 2009 / 710250$

Alquran, M. and K. Al-Khaled, 2012. Effective approximate methods for strongly nonlinear differential equations with oscillations. Math. Sci., 6: 32-32. DOI: $10.1186 / 2251-7456-6-32$

Aminikhah, H. and M. Hemmatnezhad, 2011. An effective modification of the homotopy perturbation method for stiff systems of ordinary differential equations. Applied Math. Lett., 24: 1502-1508. DOI: $10.1016 /$ j.aml.2011.03.032

Aminikhah, H., 2012. The combined Laplace transform and new homotopy perturbation methods for stiff systems of odes. Applied Math. Modell., 36: 3638-3644. DOI: 10.1016/j.apm.2011.10.014

Atay, M.T. and O. Kilic, 2013. The semianalytical solutions for stiff systems of ordinary differential equations by using variational iteration method and modified variational iteration method with comparison to exact solutions. Math. Problems Eng. DOI: $10.1155 / 2013 / 143915$

Benhammouda, B., H. Vazquez-Leal and L. HernandezMartinez, 2014. Modified differential transform method for solving the model of pollution for a system of lakes. Discrete Dynamics Nature Society. DOI: $10.1155 / 2014 / 645726$

Bervillier, C., 2012. Status of the differential transformation method. Applied Math. Comput., 218: 10158-10170. DOI: 10.1016/j.amc.2012.03.094

Butcher, J.C., 2008. Numerical methods for ordinary differential equations. 2nd Edn., John Wiley and Sons, Chichester, ISBN-10: 0470753757, pp: 482.

Chowdhury, M.S.H., I. Hashim and S. Momani, 2009. The multistage homotopy-perturbation method: A powerful scheme for handling the Lorenz system. Chaos, Solitons Fractals, 40: 1929-1937. DOI: 10.1016/j.chaos.2007.09.073

Chowdhury, M.S.H., I. Hashim, S. Momani and M.M. Rahman, 2012. Application of multistage homotopy perturbation method to the chaotic genesio system. Abstract Applied Analysis. DOI: $10.1155 / 2012 / 974293$ 
Chowdhury, M.S.H., N.I. Razali, W. Asrar and M.M. Rahman, 2014. The multistage homotopy perturbation method for solving chaotic and hyperchaotic lü system. Abstract Applied Analysis.

Darvishi, M.T., F. Khani and A.A. Soliman, 2007. The numerical simulation for stiff systems of ordinary differential equations. Comput. Math. Applic., 54: 1055-1063. DOI: 10.1016/j.camwa.2006.12.072

Djang, G.F., 1948. A modified method of iteration of the Picard type in the solution of differential equations. J. Franklin Institute, 246: 453-457. DOI: 10.1016/0016-0032(48)90261-0

Do, Y. and B. Jang, 2012. Enhanced multistage differential transform method: Application to the population models. Abstract Applied Analysis. DOI: $10.1155 / 2012 / 253890$

Domairry, G. and M. Hatami, 2014. Squeezing $\mathrm{Cu}-$ water nanofluid flow analysis between parallel plates by DTM-Padé Method. J. Molecular Liquids, 193: 37-44. DOI: 10.1016/j.molliq.2013.12.034

Ebady, A.M.N., H.M. Habib and E.R. El-Zahar, 2012. A fourth order a-stable explicit one-step method for solving stiff differential systems arising in chemical reactions. Int. J. Pure Applied Math., 81: 803-812.

Egbako, U.A. and K.R. Adeboye, 2012. One-step, sixthorder numerical method via padé approximants for the solutions of stiff differential equations. Am. J. Comput. Applied Math., 2: 10-13. DOI: $10.5923 /$ j.ajcam.20120202.02

Ehle, B.L., 1973. A-stable methods and Padé approximations to the exponential. SIAM J. Math. Analysis, 4: 671-680.

El-Zahar, E.R., 2012a. An adaptive step-size taylor series based method and application to nonlinear biochemical reaction model. Trends Applied Sci. Res., 7: 901-912.

El-Zahar, E.R., 2012b. Approximate analytical solutions for singularly perturbed boundary value problems by multi-step differential transform method. J. Applied Sci., 12: 2026-2034.

El-Zahar, E.R., 2013a. A non-linear absolutely-stable explicit numerical integration algorithm for stiff initial-value problems. Am. J. Applied Sci., 10: 1363-1363. DOI: 10.3844/ajassp.2013.1363.1370

El-Zahar, E.R., 2013b. Approximate analytical solutions of singularly perturbed fourth order boundary value problems using differential transform method. J. King Saud University-Science, 25: 257-265. DOI: 10.1016/j.jksus.2013.01.004

El-Zahar, E.R. and S.M. EL-Kabeir, 2013. A new method for solving singularly perturbed boundary value problems. Applied Math. Inf. Sci., 7: 927-938.

El-Zahar, E.R., Y.S. Hamed and H.M. Habib, 2014a. A non-linear fifth order a-stable explicit one-step method for stiff systems arising in chemical reactions. Int. J. Pure Applied Mathematics, 94: 341-354. DOI: 10.12732/ijpam.v94i3.4
El-Zahar, E.R., E.A. El-Sayed and H.M. Habib, 2014b. An application of differential transform method in singular perturbation problems. Universal J. Computat. Math., 2: 93-99.

DOI: $10.13189 /$ ujmcj.2014.020601

El-Zahar, E.R., 2015. Applications of adaptive multi step differential transform method to singular perturbation problems arising in science and engineering. Applied Math. Inf. Sci., 9: 223-232. DOI: $10.12785 / \mathrm{amis} / 090128$

Erturk, V.S., Z.M. Odibat and S. Momani, 2012. The multi-step differential transform method and its application to determine the solutions of nonlinear oscillators. Adv. Applied Math. Mechan., 4: 422-438. DOI: 10.1017/S2070073300001727

Ghosh, S., A. Roy and D. Roy, 2007. An adaptation of adomian decomposition for numeric-analytic integration of strongly nonlinear and chaotic oscillators. Comput. Methods Applied Mechan. Eng., 196: 1133-1153.

DOI: 10.1016/j.cma.2006.08.010

Gonzalez-Parra, G., A.J. Arenas and L. Jodar, 2009. Piecewise finite series solutions of seasonal diseases models using multistage adomian method. Commun. Nonlinear Sci. Numerical Simulat., 14: 3967-3977. DOI: 10.1016/j.cnsns.2009.02.023

Goh, S.M., M.S.M. Noorani and I. Hashim, 2008. Prescribing a multistage analytical method to a prey-predator dynamical system. Phys. Lett. A, 373: 107-110. DOI: 10.1016/j.physleta.2008.11.009

Gokdogan, A., M. Merdan and A. Yildirim, 2012a. Adaptive multi-step differential transformation method to solving nonlinear differential equations. Math. Comput. Modell., 55: 761-769. DOI: $10.1016 /$ j.mcm.2011.09.001

Gokdogan, A., M. Merdan and A. Yildirim, 2012b. A multistage differential transformation method for approximate solution of Hantavirus infection model. Commun. Nonlinear Sci. Numerical Simulat., 17: 1-8. DOI: 10.1016/j.cnsns.2011.05.023

Guzel, N. and M. Bayram, 2005. On the numerical solution of stiff systems. Applied Math. Comput., 170: 230-236. DOI: 10.1016/j.amc.2004.11.035

Hairer, E. and G. Wanner, 1996. Solving ordinary differential equations II: Stiff and differentialalgebraic problems. Springer-Verlag, New York.

Heydari, M., G.B. Loghmani, S.M. Hosseini and A. Yildirim, 2013. A novel Hybrid Spectral-Variational Iteration Method (H-S-VIM) for solving nonlinear equations arising in heat transfer. Iranian J. Sci. Technol. Trans. A-Sci., 37: 501-512.

Heydari, M., G.B. Loghmani and S.M. Hosseini, 2014. An improved piecewise variational iteration method for solving strongly nonlinear oscillators. Computat. Applied Math., 34: 215-249. DOI: $10.1007 / \mathrm{s} 40314-014-0113-3$ 
Innocentini, V., 1999. A successive substitution method for the evaluation of trajectories approximating the parcel path by a linear function of space and time. Mon. Wea. Rev., 127: 1639-1650.

DOI:

$10.1175 / 1520-$ 0493(1999) $127<1639:$ ASSMFT $>2.0$. CO;2

Kanth, A.S.V.R. and K. Aruna, 2013. Differential transform-padé technique for treating non-linear singular boundary value problems arising in the applied sciences. Int. J. Nonlinear Sci. Numerical Simulation, 14: 247-254. DOI: $10.1515 /$ ijnsns-2011-0005

Khader, M.M. and A.M. Megahed, 2014. Differential transformation method for studying flow and heat transfer due to stretching sheet embedded in porous medium with variable thickness, variable thermal conductivity and thermal radiation. Applied Math. Mechanics, 35: 1387-1400.

DOI: $10.1007 / \mathrm{s} 10483-014-1870-7$

Keimanesh, M., M.M. Rashidi, A.J. Chamkha and R. Jafari, 2011. Study of a third grade non-Newtonian fluid flow between two parallel plates using the multi-step differential transform method. Computers Math. Applications, 62: 2871-2891.

DOI: $10.1016 /$ j.camwa.2011.07.054

Lal, M. and D. Moffatt, 1982. Picard's successive approximation for non-linear two-point boundaryvalue problems. J. Computational Applied Mathematics, 8: 233-236. DOI: $10.1016 / 0771-050 \mathrm{X}(82) 90045-6$

Lambert, J.D., 1991. Numerical methods for ordinary differential systems: the initial value problem. 1st Edn., Wiley, Chichester, ISBN-10: 0471929905, pp: 293.

Lee, C.H., K.D. Park and B. Jang, 2013. Multistage homotopy perturbation method for nonlinear reaction networks. J. Mathematical Chemistry, 51: 1945-1960. DOI: 10.1007/s10910-013-0195-y

Lu, J., 2012. The GDTM-Padé Technique for the Nonlinear Lattice Equations. Abstract Applied Analysis, DOI: 10.1155/2012/863583

Mahmood, A.S., L. Casasus and W. Al-Hayani, 2005. The decomposition method for stiff systems of ordinary differential equations. Applied Math. Computat., 167: 964-975. DOI: 10.1016/j.amc.2004.06.134

Molli, R.Y., M.S.M. Noorani, R.R. Ahmad and A.K. Alomari, 2013. A step variational iteration method for solving non-chaotic and chaotic systems. Sains Malaysiana, 42: 347-358.

Momani, S., A. Freihat and M. Al-Smadi, 2014. Analytical study of fractional-order multiple chaotic Fitzhugh-Nagumo neurons model using multistep generalized differential transform method. Abstract Applied Analysis. DOI: 10.1155/2014/276279

Nik, H.S. and F. Soleymani, 2013. A Taylor-type numerical method for solving nonlinear ordinary differential equations. Alexandria Eng. J., 52: 543-550. DOI: 10.1016/j.aej.2013.02.006
Novati, P., 2003. An explicit one-step method for stiff problems. Computing, 71: 133-151. DOI: $10.1007 / \mathrm{s} 00607-003-0021-7$

Odibat, Z.M., C. Bertelle, M.A. Aziz-Alaoui and G.H. Duchamp, 2010. A multi-step differential transform method and application to non-chaotic or chaotic systems. Comput. Math. Applic., 59: 1462-1472. DOI: 10.1016/j.camwa.2009.11.005

Olvera, D., A. Elias-Zuniga, L.N. Lopez de Lacalle and C.A. Rodriguez, 2014. Approximate solutions of delay differential equations with constant and variable coefficients by the enhanced multistage homotopy perturbation method. Abstract Applied Analysis, DOI: 0.1155/2015/382475

Patra, A. and S.S. Ray, 2013. Multistep differential transform method for numerical solution of classical neutron point kinetic equation. Computat. Math. Modell., 24: 604-615. DOI: $10.1007 / \mathrm{s} 10598-013-9202-1$

Rashidi, M.M. and S.A. Mohimanian Pour, 2010a. A novel analytical solution of heat transfer of a micropolar fluid through a porous medium with radiation by DTM-Padé. Heat Transfer-Asian Research, 39: 575-589. DOI: 10.1002/htj.20317

Rashidi, M.M. and M. Keimanesh, 2010b. Using differential transform method and Padé approximant for solving MHD flow in a laminar liquid film from a horizontal stretching surface. Math. Problems Eng. DOI: $10.1155 / 2010 / 491319$

Rashidi, M.M., T. Hayat, T. Keimanesh and H. Yousefian, 2013. A study on heat transfer in a second-grade fluid through a porous medium with the modified differential transform method. Heat Transfer-Asian Res., 42: 31-45. DOI: 10.1002/htj.21030

Rashidi, M.M., A.B. Parsa, O.A. Bég, L. Shamekhi and S.M. Sadri et al., 2014. Parametric analysis of entropy generation in magneto-hemodynamic flow in a semi-porous channel with OHAM and DTM. Applied Bionics Biomechanics. 11: 47-60. DOI: $10.3233 / \mathrm{ABB}-140086$

Schiesser, W.E., 1993. Computational Mathematics in Engineering and Applied Science: ODEs, DAEs and PDEs. 1st Edn., CRC Press, ISBN-10: 0849373735, pp: 608.

Shyy, W., M.H. Chen, R. Mittal and H.S.U. Kumar, 1992. On the suppression of numerical oscillations using a non-linear filter. J. Computational Physics, 102: 49-62.

DOI: 10.1016/S0021-9991(05)80004-X

Smarda, Z., J. Diblik and Y. Khan, 2013. Extension of the differential transformation method to nonlinear differential and integro-differential equations with proportional delays. Adv. Difference Equ. DOI: 10.1186/1687-1847-2013-69 
Vulanovic, R., 1989. A uniform numerical method for quasilinear singular perturbation problems without turning points. Computing, 41: 97-106.

DOI: $10.1007 / \mathrm{BF} 02238732$

Wang, S., Y. Yu and M. Diao, 2010. Application of multistage homotopy-perturbation method in hybrid synchronization of chaotic systems. Int. J. Comput. Math., 87: 3007-3016.

DOI: $10.1080 / 00207160902874661$

Wazwaz, A.M., 2011. Linear and nonlinear integral equations: methods and applications. Springer. DOI: $10.1007 / 978-3-642-21449-3$

Wu, X.Y., 1998. A sixth-order A-stable explicit one-step method for stiff systems. Comput. Math. Applic., 35: 59-64. DOI: 10.1016/S0898-1221(98)00057-1

$\mathrm{Wu}, \mathrm{X} . \mathrm{Y}$. and J.L. Xia, 2001. Two low accuracy methods for stiff systems. Applied Math Computation, 123: 141-153.

DOI: 10.1016/S0096-3003(00)00010-2

$\mathrm{Wu}, \mathrm{X}$. and J. Xia, 2007. Study of general taylor-like explicit methods in solving stiff ordinary differential equations. Int. J. Comput. Math., 84: 1795-1803. DOI: $10.1080 / 00207160701331400$

Yildirim, A., A. Gokdogan and M. Merdan, 2012. Chaotic systems via multi-step differential transformation method. Canadian J. Phys., 90: 391-406.

DOI: $10.1139 / \mathrm{p} 2012-032$
Youssef, I.K. and H.A. El-Arabawy, 2007. Picard iteration algorithm combined with Gauss-Seidel technique for initial value problems. Applied Math. Comput., 190: 345-355.

DOI: $10.1016 /$ j.amc.2007.01.058

Yost, S.A. and P. Rao, 2000. A non-linear filter for oneand two-dimensional open channel flows with shocks. Adv. Water Resources, 24: 187-193. DOI: 10.1016/S0309-1708(00)00038-5

Zhou, J.K., 1986. Differential Transformation and its Applications for Electrical Circuits. 1st Edn., Huazhong University Press, Wuhan, China (Chinese).

Zou, L., Z. Wang, Z. Zong, D.Y. Zou and S. Zhang, 2012. Solving shock wave with discontinuity by Enhanced Differential Transform Method (EDTM). Applied Math. Mechan., 33: 1569-1582. DOI: $10.1007 / \mathrm{s} 10483-012-1644-9$

Zhao, Y. and A. Xiao, 2010. Variational iteration method for singular perturbation initial value problems. Comput. Phys. Commun., 181: 947-956. DOI: $10.1016 /$ j.cpc.2010.01.007

Zhao, Y., A. Xiao, L. Li and C. Zhang, 2014. Variational iteration method for singular perturbation initial value problems with delays. Math. Problems Eng. DOI: $10.1155 / 2014 / 850343$ 